\title{
DETERMINANTES DE LA COHESIÓN EN LA VOTACIÓN DEL LEGISLATIVO EN LAS COALICIONES OFICIALISTAS Y DE OPOSICIÓN EN LA CÁMARA DE DIPUTADOS DE CHILE, 2006-2014 ${ }^{1}$ \\ Determinants of Cohesion in the Legislative Voting of Government and Opposition Coalitions in the Chilean Chamber of Deputies, 2006-2014
}

\author{
DAVID MARTÍNEZ \\ Universidad Diego Portales \\ davidsebas.martinez@gmail.com \\ PATRICIO NAVIA \\ Universidad Diego Portales \\ New York University \\ pdn200@nyu.edu \\ Cómo citar/Citation \\ Martínez, D. y Navia, P. (2019). \\ Determinantes de la cohesión en la votación del legislativo en las coaliciones \\ oficialistas y de oposición en la Cámara de Diputados de Chile, 2006-2014. \\ Revista de Estudios Políticos, 185, 225-258 \\ doi: https://doi.org/10.18042/cepc/rep.185.08
}

\section{Resumen}

Si los incentivos institucionales son los mismos y la cohesión partidista en las votaciones del Legislativo varía dependiendo del partido que controla el poder, podemos concluir que la cultura partidista afecta la cohesión del contingente legislativo de un partido tanto cuando es Gobierno como cuando es oposición. Entre 1990 y 2010,

Agradecemos los comentarios de los dos revisores anónimos y el financiamiento de la Comisión Nacional de Investigación Científica y Tecnológica de Chile [CONICYT/ FONDAP/15130009] y del Fondo Nacional de Desarrollo Científico y Tecnológico [FONDECYT REGULAR 1171051]. 
la centroizquierdista Concertación controló la Presidencia y (excepto en 20082009) la mayoría en la Cámara de Diputados de Chile. Entre 2010 y 2014, la centroderechista Alianza y sus aliados controlaron la Presidencia y la mayoría en la Cámara de Diputados. Con datos de 5216 roll call votes en los dos periodos legislativos entre 2006 y 2014, analizamos la cohesión en la votación en la Cámara de Diputados en las coaliciones oficialista y de oposición. Evaluamos dos hipótesis. Reportamos que el oficialismo está más cohesionado que la oposición y que ambas coaliciones se cohesionan más en votaciones más importantes. Adicionalmente, mostramos que la Concertación tiende a cohesionarse más como coalición de Gobierno hacia el final del periodo, mientras que la Alianza lo hace menos. Bajo las mismas reglas, la izquierda y la derecha se comportan de forma distinta como oficialismo y oposición.

\section{Palabras clave}

Unidad partidaria; cohesión partidaria; votaciones legislativas; cuórums legislativos; proceso legislativo; Cámara de Diputados; Chile.

\section{Absctract}

When institutional incentives are equal and cohesion in the legislative behaviour of political parties varies depending on which of them hold power, we can assume that Party culture affects the level of voting cohesion of its legislative delegation, whether in government or in opposition. Between 1990 and 2010, the centre-left Concertación coalition controlled the presidency and (except for 2008-2009) the majority in the Chilean Chamber of Deputies. Between 2010 and 2014, the centre-right Alianza and its allies controlled the presidency and the majority in the Chamber of Deputies. With data from 5,216 roll-call votes in the two legislative terms between 2006 and 2014, we analyse cohesion in the voting behavior of legislators in the government and opposition coalitions. We assess two hypotheses, finding that the governing coalition is more cohesive than the opposition and that both coalitions are more disciplined for roll call votes that are of greater importance. We also report that Concertación becomes more cohesive and Alianza less so toward the end of presidential terms. Under the same legislative rules, then, the left and right coalitions behave differently in terms of their voting cohesiveness.

\section{Keywords}

Party unity; roll call votes; party discipline; legislative quorums; legislative process; Chamber of Deputies; Chile. 


\section{SUMARIO}

I. DETERMINANTES DE LA UNIDAD PARTIDISTA EN LAS VOTACIONES. II. DETERMINANTES DE LA UNIDAD PARTIDISTA EN EL SISTEMA POLÍTICO CHILENO. III. METODOLOGÍA. IV. ANÁLISIS DESCRIPTIVO. V. ANÁLISIS INFERENCIAL. VI. CONCLUSIÓN. BIBLIOGRAFÍA.

La cohesión partidista en el comportamiento legislativo es afectada por variables de diseño institucional, pero la cultura partidista también pudiera influir sobre la cohesión de los legisladores de distintos partidos al votar proyectos de ley cuando son Gobierno y cuando son oposición. Para evaluar esto, utilizamos el caso de la Cámara de Diputados en Chile entre 2006 y 2014. Con datos de 5216 roll call votes (RCV) en el periodo 2006-2014 - un cuatrienio cuando la centroizquierdista Concertación ejerció el poder y el otro bajo un Gobierno de la centroderechista Alianza - evaluamos la cohesión partidista de las coaliciones oficialistas y de oposición para diferenciar entre el efecto de las reglas institucionales y de cultura partidista.

Entre las variables de diseño institucional, evaluamos el efecto del trámite legislativo, tipo de cuórum del calendario electoral, de la condición de mayoría y de la ideología sobre la cohesión de las bancadas de las coaliciones oficialista y de oposición. Analizamos dos hipótesis. Primero, que el oficialismo está más cohesionado que la oposición. Segundo, que la cohesión legislativa aumenta cuando se votan proyectos que requieren de cuórums más altos y en trámites legislativos más avanzados, y que mientras más importante sea la votación, hay mayor cohesión en la coalición oficialista que en la oposición.

En lo que sigue, primero discutimos los determinantes de la unidad partidista en las votaciones de los legisladores en general y de la cohesión partidaria en las votaciones del contingente legislativo del partido en particular. Después, presentamos la evolución del sistema de partidos y los determinantes del sistema electoral y del sistema político chileno que han inducido a la formación de coaliciones estables desde 1990, y en particular entre 2006 y 2014. También explicitamos nuestras hipótesis. Posteriormente, justificamos la metodología utilizada, explicamos el uso de los RCV y los indicadores WUnity y WRice para medir la cohesión partidista, y exponemos los resultados electorales y la composición de la Cámara de Diputados por partidos políticos y coaliciones. En cuarto lugar, con datos de 5216 RCV en el periodo, evaluamos las hipótesis. Finalmente, entregamos nuestras conclusiones. 


\section{DETERMINANTES DE LA UNIDAD PARTIDISTA EN LAS VOTACIONES}

Las explicaciones sobre la formación y funcionamiento de los sistemas de partidos identifican como variables explicativas a los clivajes societales (Lipset y Rokkan, 1967) o las instituciones y reglas electorales (Duverger, 1954). Contribuciones posteriores han destacado tanto la interacción entre ambos grupos de determinantes (Lijphart; 1990; Grofman y Lijphart, 1986; Sartori, 1986) y han especificado las relaciones de causalidad entre los incentivos que entregan los clivajes sociales y las leyes electorales (Cox, 1997; Colomer, 2005). Como es evidente que las leyes electorales importan, pero hay amplia evidencia de que los clivajes sociales —o la cultura política- de un país también influyen en el comportamiento de los partidos, resulta importante aislar los efectos de las reglas del juego político de aquellos que responden a tradiciones y culturas partidistas. Por ejemplo, podemos identificar si los partidos de izquierda responden de forma distinta que los partidos de derecha a los incentivos institucionales y si los partidos de izquierda y de derecha se comportan de forma distinta cuando están en el poder que cuando son oposición. Cuando se aplican estas lógicas a la cohesión en las votaciones de sus legisladores en el Congreso, si no hay diferencias entre partidos de izquierda y de derecha, la unidad partidista respondería más bien a las reglas del juego. Si en cambio los partidos de izquierda se comportan de forma distinta que los partidos de derecha cuando son oposición que cuando son Gobierno, la unidad partidista se explicaría por cultura partidista o cohesión ideológica.

Para analizar el comportamiento de los contingentes partidistas en el poder legislativo, también resulta clave entender la estructura de la relación entre los poderes ejecutivo y legislativo (Shugart, 2008). En sistemas presidenciales, los incentivos se generan a partir de la elección popular tanto del presidente como de los congresistas y, por tanto, existen incentivos para cooperar en el proceso de formación de leyes (ibid.: 346). Cuando los sistemas presidenciales coexisten con legislaturas electas con sistemas de representación proporcional y ambas elecciones son concurrentes, a menudo los presidentes deben acudir a la formación de coaliciones pre o postelectorales, en tanto el contingente legislativo de sus partidos no es suficiente para ejercer control mayoritario del Congreso (Golder, 2006). En algunos países, esta combinación ha llevado a la formación de coaliciones multipartidistas estables (Carey, 2009), mientras que en otros ha sido sindicada como causa de la inestabilidad del presidencialismo (Linz y Valenzuela, 1994).

La cooperación entre el ejecutivo y el legislativo está determinada por las reglas del juego que pueden darle poderes y atribuciones legislativas al 
ejecutivo y que, por lo tanto, especialmente en América Latina, reducen los poderes formales del legislativo (Morgenstern y Nacif, 2002). Con todo, incluso en sistemas altamente presidencialistas, para avanzar en sus agendas legislativas los presidentes deben construir mayorías en el Congreso (Figueiredo y Limongi, 2000). Como el diseño institucional en sistemas presidencialistas puede dar al ejecutivo poderes y atribuciones para influir en la agenda legislativa, en muchos casos estos instrumentos les facilitan la negociación con el poder legislativo (Cox y McCubbins, 2005: 20-25). De ahí que la cohesión en el comportamiento del legislativo se explique, al menos en parte, por los poderes y atribuciones del ejecutivo para influir sobre la agenda legislativa y sobre el comportamiento de los legisladores que pertenecen a su partido o coalición de Gobierno. Mientras más atribuciones tiene el ejecutivo, más poder debiese tener para lograr que su contingente legislativo se discipline en torno a sus prioridades. Luego, un mismo partido debiera demostrar distintos niveles de cohesión en su comportamiento cuando es parte de una coalición de Gobierno que cuando está en la oposición.

A su vez, el poder legislativo puede organizarse de tal forma de convertirse en una contraparte efectiva para el poder ejecutivo. Ya sea porque los legisladores se convierten en un cartel para solucionar los problemas de acción colectiva que implica la organización eficiente del Congreso o porque se ordenan en torno a su militancia partidista para construir coaliciones mínimas ganadoras que les permitan controlar el Congreso, independientemente de quién controla el ejecutivo. Luego, las reglas del juego que regulan el funcionamiento del congreso constituyen incentivos o desincentivos para que los legisladores puedan forjar coaliciones y acuerdos entre ellos, tanto en la forma en que negocian con el ejecutivo, como en la formación de mayorías para controlar el funcionamiento del Congreso.

Independientemente de si militan en partidos o son independientes, los legisladores actúan bajo dos lógicas, buscando la producción de políticas y promoviendo su reelección o carreras futuras (Cox, 2006; Shepsle y Weingast, 1994). Dependiendo de las reglas de cada país, la reelección de los congresistas puede estar condicionada por la participación de líderes partidarios en la designación de las listas para competir en la próxima elección. De ahí que la ambición de cada congresista está limitada por el poder que poseen los líderes de sus partidos respectivos. Los sistemas políticos que poseen listas cerradas están más cohesionados que aquellos sistemas con listas abiertas. Lo anterior se debe a que los líderes partidarios participan en la designación de los candidatos en las listas cerradas y los congresistas tienen un comportamiento responsivo hacia el líder de partido, no así cuando hay listas abiertas y los políticos desarrollan bases de apoyo personales y se genera competencia intrapartidaria (Carey, 2007). 
En las bancadas de gran tamaño, los legisladores poseen una serie de beneficios que inducen a la cohesión en su comportamiento (Carey, 2009). Los beneficios se pueden clasificar en asignaciones de comités y en recursos y personal que pueden ser utilizados para el trabajo distrital o el trabajo en campañas. Conjuntamente, cuando las bancadas deciden cómo se efectuará el voto de los congresistas, se facilita la construcción de consensos y la cohesión del contingente partidista en el Congreso y, cuando son bancadas oficialistas, la concreción del programa de gobierno. Carey (ibid.) recuerda que los líderes partidarios imponen sanciones a legisladores que quiebran con la cohesión en la bancada.

El diseño institucional ayuda a explicar la unidad o cohesión partidista en las votaciones que se realizan en el Congreso (Morgenstern, 2004). Este diseño institucional facilita la cohesión que "puede resultar desde sanciones o de incentivos positivos para que los congresistas voten en conjunto incluso cuando sus preferencias difieran» (Sieberer, 2006: 162). Por eso, la «disciplina se refiere a la combinación de zanahorias y garrotes, generalmente administrado por los líderes partidarios, usados para recompensar la lealtad cuando se vota y de mantener o castigar los actos de disciplina» (Carey, 2007: 93). Los partidos constituyen un leviatán legislativo que coerce el actuar individual de los congresistas para avanzar en la agenda programática de la coalición del Gobierno (Cox y McCubbins, 2007).

Estudios anteriores han demostrado que los líderes de los partidos políticos ordenan a su bancada en el Congreso al momento de las votaciones (Carey, 2009; Poole y Rosenthal, 2000). Özbudun (1970) sostiene que la unidad partidista en el comportamiento en votaciones en el Congreso se explica por la cohesión ideológica de los miembros o por la disciplina que ejerce el liderazgo partidista sobre los miembros (Morgenstern, 2004). Sin embargo, los valores y actitudes de los legisladores y las características de los partidos a los que pertenecen influyen también sobre la cohesión en el comportamiento legislativo (Londregan, 2000; Toro Maureira, 2007). Al enfocar su estudio en la dimensión de lealtad partidaria, Close (2018) señala que esta deriva de la asunción por parte de los legisladores de las normas de unidad partidaria y de solidaridad y que «lleva a los parlamentarios a apegarse a la línea del partido incluso en caso de desacuerdo" (Close, 2018: 212). Utilizando datos de catorce legislaturas de Europa, Close muestra que el tipo de familia partidaria - ordenada en una escala de siete valores, desde la izquierda radical a derecha radical, que, por cierto, se diferencia de la ideología partidista - también afecta la cohesión partidaria.

No sorprende entonces que las investigaciones que abordan la unidad partidaria a menudo identifican un efecto combinado de elementos de diseño institucional e incentivos formales e informales con los procedimientos y 
normas que desarrollan los propios partidos para solucionar los problemas de acción colectiva que enfrentan los legisladores que necesitan avanzar sus propias carreras, pero que también buscan, como partido, ejercer control colectivo mayoritario en el Congreso (Carey, 2007; Shepsle y Weingast 1994; Cox y McCubbins, 2007, 2005).

En América Latina, los presidencialismos presentan poderes reactivos y proactivos para mantener o cambiar el statu quo respectivamente, tal como en Chile (Linz y Valenzuela, 1998; Mainwaring y Shugart, 2002). Entre los poderes proactivos, destacan los poderes legislativos del presidente que le permiten influir en el comportamiento de los congresistas en la votación de las leyes. Estudios sobre cohesión legislativa en América Latina sostienen que la coalición oficialista está más cohesionada en sus votaciones que la coalición opositora. Algunos reportan que la unidad partidista varía dependiendo de las reglas electorales, aunque otros también han reportado que esta depende, al menos en parte, de la cultura de los partidos tanto como de las reglas electorales (Aleman y Calvo, 2008; Alemán, 2006). Como las características constitucionales del sistema político influyen en los niveles de cohesión de los congresistas, debemos entender cuáles son las reglas que rigen el caso de estudio para elaborar las hipótesis que permitan verificar si los incentivos influyen sobre el comportamiento de los partidos en las votaciones en el congreso.

\section{DETERMINANTES DE LA UNIDAD PARTIDISTA EN EL SISTEMA POLÍTICO CHILENO}

$\mathrm{Al}$ igual que otros países en América Latina, Chile posee un sistema político fuertemente presidencialista. Desde el retorno de la democracia en 1990, el sistema político ha estado caracterizado por coaliciones estables y permanentes formadas por partidos políticos que han mantenido su autonomía e independencia. La política se ordena en torno a dos coaliciones, la centroizquierdista Concertación y la centroderechista Alianza. Hay al menos siete partidos fácilmente identificables. De izquierda a derecha, han obtenido representación política en distintos tipos de puestos de elección popular, el Partido Comunista (PC), Partido Socialista (PS), Partido por la Democracia (PPD), Partido Radical (PR), Partido Demócrata Cristiano (PDC) - todos ellos como miembros de la Concertación - y Renovación Nacional (RN) y Unión Demócrata Independiente (UDI), como miembros de la Alianza. Si bien existen y han existido otros partidos que también han tenido representación política en el Congreso, estos siete partidos agrupan a la gran mayoría de los legisladores electos en Chile entre 1989 y 2014. 
Posteriormente al golpe militar, se argumenta que existe un nuevo clivaje originado por la transición a la democracia que se expresa en la formación de coaliciones electorales (Tironi y Agüero, 1999). En contra de esta argumentación, se señala que el sistema electoral binominal obliga a los partidos políti$\cos$ a conformar coaliciones electorales, pero que la cultura política multipartidista sobrevivió (Siavelis, 2002). Si los partidos políticos no se integran en las coaliciones, les resulta difícil aspirar a obtener un escaño legislativo, por lo que el sistema electoral binominal actúa como una camisa de fuerza que inhibe la reaparición del sistema multipartidista (Valenzuela, 1999). Por esto, la Concertación y la Alianza se configuraron como las coaliciones predominantes en el sistema de partidos (Siavelis, 2000; Londregan, 2000; Siavelis, 2005; Carey, 2006). En resumen, un sistema electoral presidencial de segunda vuelta y un sistema electoral binominal en las elecciones legislativas inducen a la formación de coaliciones e inhiben la aparición multipartidista.

Varios estudios han reportado los amplios poderes legislativos del presidente chileno (Siavelis, 2000) y las limitantes que tiene el poder legislativo para convertirse en un contrapeso efectivo (Baldez y Carey, 1999), pero estudios posteriores han destacado la capacidad de bloqueo y negociación que tiene el poder legislativo para limitar las prerrogativas del ejecutivo (Aninat $e t$ al., 2008; Aninat, 2006; Toro Maureira et al., 2011). El poder legislativo tiene capacidad de obstruir al ejecutivo e influir sobre las leyes que se promulgan (Aninat et al., 2008). De hecho, aunque los partidos oficialistas tuviesen minoría en el Congreso, en la medida que sea capaz de lograr acuerdos con los partidos de oposición, o al menos con algunos legisladores de oposición, el presidente puede avanzar su agenda legislativa. A su vez, aún si los partidos de la coalición de Gobierno tienen el control mayoritario del Congreso, el presidente no podrá avanzar su agenda a menos de que logre un alto nivel de cohesión entre los legisladores (Aninat et al., 2006; Aleman y Saiegh, 2007; Campos-Parra y Navia, 2017).

El Ejecutivo en Chile es un colegislador privilegiado. El presidente posee poderes proactivos que se identifican en la iniciativa de ley del Ejecutivo e ingresar las urgencias — un mecanismo que establece prioridad en la agenda legislativa - a los proyectos de ley que presenta. El presidente puede hacer uso de una urgencia simple, suma urgencia y discusión inmediata en una de las Cámaras, lo que implica que esta deba tramitar el proyecto en treinta días, diez días o tres días respectivamente (estos platos se modificaron a 30, 15 y 6 respectivamente en 2010). Además, el Ejecutivo cuenta con el veto presidencial que se presenta de dos formas. Primero, puede ser un veto modificatorio que altera el contenido de algunos artículos de un proyecto de ley. Segundo, puede realizar un veto absoluto a un proyecto de ley que implica un rechazo a 
la totalidad del proyecto de ley. En ambos casos, para revertir la decisión del Ejecutivo, el Congreso requiere de una mayoría de dos tercios de sus miembros. No obstante, aunque es uno de los presidencialismos más exacerbado de la región, la evidencia también muestra que existe cooperación entre el Ejecutivo y el Legislativo, lo que induce a una mayor productividad legislativa (Aninat et al., 2008). En el caso de no producirse cooperación, el presidente tiene la facultad de convocar plebiscitos en proyectos de ley con temática de reforma constitucional (Aninat, 2006).

Apuntando a los incentivos institucionales, Aleman y Saiegh (2007) destacan que los legisladores se ordenan en torno a las coaliciones a las que pertenecen, mientras que Aleman y Navia (2009) destacan los poderes legislativos del presidente, especialmente en periodos de luna de miel, para dar cuenta de la cohesión en el comportamiento partidista en el congreso chileno. De forma similar, Toro Maureira (2007) muestra que los poderes legislativos del Ejecutivo se favorecen por la férrea disciplina partidista de las coaliciones, aunque también muestra diferencias en el interior de las coaliciones dependiendo del partido en el que militan los legisladores. Por otro lado, Visconti (2011) muestra que los legisladores van desarrollando un expertise que los lleva, a medida que pasan los años, a reducir el desbalance de poderes a favor del Ejecutivo. De hecho, el Ejecutivo necesita coordinarse con el Poder Legislativo para poder avanzar su agenda (Toro-Maureira y Hurtado, 2016), pero hay evidencia reciente que muestra que el comportamiento del Legislativo varía dependiendo de qué coalición está en el poder (Campos-Parra y Navia, 2017).

Entre los estudios sobre cohesión en la votación de los legisladores chilenos, Carey (2002) sostiene que el comportamiento legislativo se explica tanto por incentivos institucionales como por las dinámicas de alianzas partidistas y por el control que ejercen los liderazgos de los partidos sobre el comportamiento de los legisladores. Si bien reconoce la importancia de la ideología, Londregan (2000), en un estudio sobre el funcionamiento del Senado a comienzos de los noventa, muestra el efecto que tienen las reglas para explicar el comportamiento de los legisladores. A su vez, Campos-Parra y Navia (2017) destacan que la mayor disciplina que se observó en el comportamiento de la centroizquierdista Concertación cuando fue Gobierno fue también observada en la centroderechista Alianza cuando esa coalición fue Gobierno.

Como las elecciones legislativas y presidenciales en Chile se realizan cada cuatro años de forma concurrente desde 2006, las dinámicas que afectan la política a nivel de presidente influyen también en el comportamiento legislativo. Cuando el presidente recién ha asumido el poder, tiene más capacidad de inducir disciplina en su coalición (Alemán y Navia, 2009), por lo que, presumiblemente, cuando se acerca el fin del periodo ese nivel de disciplina debiese disminuir. 
Ahora bien, como la Concertación gobernó Chile entre 1990 y 2010, muchas de las conclusiones a las que llegaron estudios realizados en la época sobre el comportamiento del Legislativo y la influencia del Ejecutivo sobre dicho comportamiento sufrían de un sesgo de selección. Cuando la centroderechista Alianza llegó al poder en 2010-2014, recién se pudo evaluar si los patrones identificados para el periodo concertacionista se reproducirían también en un Gobierno en que el Ejecutivo era controlado por la coalición de derecha. Resulta lógico esperar, por ejemplo, que, en su primer Gobierno desde el retorno de la democracia, un presidente de derecha debiera inducir mayor capacidad de disciplina en los legisladores de su coalición de la que ejercieron los presidentes de centroizquierda después de terminado el primer periodo democrático (1990-1994).

Para evaluar el efecto de los incentivos institucionales y de la cultura partidista sobre la cohesión en el comportamiento partidista, postulamos 2 hipótesis para el caso de Chile:

H1. El oficialismo muestra mayor cohesión en su comportamiento. No hay diferencias si el oficialismo es la derechista Alianza o la centroizquierda Concertación.

H2. Hay más cohesión partidista en el comportamiento legislativo cuando el cuórum es más alto y el trámite legislativo es más avanzado, y esa cohesión es mayor en el oficialismo que en la oposición.

\section{METODOLOGÍA}

Los estudios sobre unidad partidista en el comportamiento legislativo normalmente utilizan como variable de análisis los votos en sala, los RCV. Los RCV son un indicador confiable para la medición del comportamiento legislativo (Rice, 1925; Clausen, 1967; Grumm, 1963; Morgenstern, 2004). En Estados Unidos, los estudios sobre el comportamiento del Congreso utilizan ampliamente este indicador (Poole, 2005; Covington et al., 1995; Ansolabehere y Jones, 2010; Poole y Rosenthal, 2000). También lo usan estudios sobre el comportamiento legislativo en América Latina (Aleman y Saiegh, 2007; Calvo, 2007; Morgenstern, 2004) y en Chile (Toro Maureira, 2007; Campos-Parra y Navia, 2017; Toro-Maureira y Hurtado, 2016).

Recolectamos los RCV de los ciento veinte diputados en ejercicio en las 5216 votaciones en sala entre el 11 de marzo de 2006 y el 11 de marzo de 2014 para los dos periodos legislativos que coinciden con las presidencias de la centroizquierdista Michelle Bachelet (2006-2010) y del centroderechista Sebastián Piñera (2010-2014). 
Nuestra variable dependiente es la unidad en la votación partidista en cada RCV. Los datos de las 2027 votaciones durante el Gobierno de Bachelet (2006-2010) fueron inicialmente recolectados por el Programa Auditoria de la Democracia del PNUD en Chile. Esa base de datos está públicamente disponible previa petición al PNUD. Obtuvimos esa base de datos y corregimos algunos errores que encontramos con la información públicamente disponible en la página web de la Cámara de Diputados del Congreso Nacional $^{2}$. A su vez, utilizamos la información de los boletines de los proyectos de ley en la página web de la Cámara para recopilar las 3189 votaciones durante el cuatrienio de Piñera. Así tenemos un total de 5216 RCV en esos ocho ańos.

La Cámara estaba compuesta por ciento veinte diputados. Cuando algún legislador dejó su escaño durante el periodo, su remplazante fue nombrado por su partido. De ahí que en cada votación solo pudieron votar, como máximo, ciento veinte diputados. Consideramos a cada diputado como parte del partido en el que fueron electos para todo el periodo, independientemente de si renunciaron a sus partidos y/o bancadas en algún momento del periodo. Aquellos independientes electos patrocinados por partidos o coaliciones son considerados como militantes de esos partidos.

Utilizamos los dos indicadores más comunes para medir cohesión o unidad partidaria, WRice y WUnity. Siguiendo a Carey (2009: 126), usamos los términos cohesión y unidad de forma intercambiable. Ambos conceptos pudieran ser entendidos de forma distinta. Cohesión es la similitud en la forma en que votan los miembros de un mismo partido, mientras que disciplina es la capacidad de los líderes del partido para lograr imponer un comportamiento determinado entre sus legisladores. Si siguiéramos esa distinción, en rigor, solo estamos evaluando cohesión o unidad y no disciplina.

WUnity y WRice miden la cohesión de 0 (nula cohesión) a un 1 (alta cohesión) teniendo como propósito determinar quién votó con quién, hasta qué grado y cómo se comportan los partidos y coaliciones. La diferencia entre ambas puntuaciones radica en que WUnity calcula todas las acciones, incluyendo abstenciones e inasistencias. En cambio, WRice solamente calcula quienes se presentan a votar. Para estimar los indicadores, seguimos a Carey (Carey, 2007, 2009). El índice WRice se construye de la siguiente forma:

$$
\text { WRice }=\frac{\sum \text { RICEij } * \text { ATTENDj } * \text { CLOSEj }}{\sum \text { ATTENDj } * \text { CLOSEj }}
$$

2 Disponible en: https://www.camara.cl/trabajamos/sala_votaciones.aspx 
- Donde:

ATTEND $j=\%$ de legisladores que votaron en un tema $j$

CLOSSEj $=1-\% S I-\% \mathrm{NO}$, para todos los legisladores que votaron en un tema $\mathrm{j}$.

RICEij: \%SI - \% NO, con la coalición o partido i en el tema j.

El índice WUnity del siguiente modo:

WUnity $=\frac{\sum \text { UNITYij } * \text { ATTEND } * \text { CLOSEj }}{\sum \text { ATTENDj } * \text { CLOSEj }}$

- Donde:

ATTEND $j=\%$ de legisladores que votaron en un tema $j$

CLOSSEj $=1-\% S I-\% N O$, para todos los legisladores que votaron en un tema $\mathrm{j}$.

UNITYij: \% SI - \%NO, con la coalición o partido i en el tema j.

WRice no castiga la unidad de un partido cuando algún legislador se ausenta o abstiene. Por el contrario, WUnity contabiliza el total de congresistas del partido, considerando las ausencias y abstenciones como votaciones en contra de la forma en que votó mayoritariamente ese partido — esto para incorporar las ausencias que se producen cuando, de manera estratégica, los congresistas se ausentan o abstienen para no votar contra sus partidos-. Por eso, el índice WRice siempre va a ser mayor que WUnity.

Nuestras variables independientes son institucionales y culturales. Las culturales son la militancia partidista de los diputados (a nivel de partido y coalición) y la condición de oficialismo u oposición de cada coalición. Las institucionales son el momento en el periodo presidencial en que se realiza la votación, el cuórum requerido para aprobar el proyecto de ley al que pertenece cada votación y el estado del trámite constitucional de cada proyecto de ley.

La Constitución de la República (CPR) jerarquiza cuatro tipos de cuórums: leyes interpretativas a la Constitución, leyes orgánicas constitucionales (LOC), leyes de cuórum calificado y cuórum simple. Las leyes de cuórum simple requieren una mayoría absoluta de los diputados presentes en sala; los cuórums interpretativos requieren una mayoría de dos tercios de todos los diputados (80 votos) o de tres quintos (72 diputados); las LOC requieren mayoría de cuatro séptimos (69 diputados); las de cuórum calificado mayoría absoluta (61 diputados), y las leyes de cuórum simple requieren mayoría de los diputados presentes.

Identificamos tres etapas en el proceso legislativo. El primer trámite constitucional se realiza en la Cámara de origen de un proyecto y contiene una discusión en general, con un informe previo discutido por la Comisión 
respectiva, pudiendo resultar en la aprobación o rechazo de la idea de legislar. En caso de aprobación, el proyecto se remite nuevamente a la Comisión para ser estudiado en particular, pudiendo resultar en indicaciones por parte de congresistas o del Poder Ejecutivo. Una vez aprobada por la Comisión, el proyecto de ley se discute en particular en la Sala pudiendo resultar en aprobación o rechazo. En caso de que se apruebe, pasa a segundo trámite constitucional en la Cámara revisora y pasa por las mismas etapas. En caso de rechazo en la Cámara revisora, no se puede legislar por la misma materia dentro de un año. En caso de la aprobación en particular por parte de la Cámara revisora del proyecto de ley tal como ingresó se convierte en ley. En caso de rechazo o modificaciones a lo aprobado por la Cámara de origen se procede al tercer trámite constitucional debiendo ser tramitada en la Cámara de origen.

En caso del tercer trámite constitucional, ambas Cámaras nominan cinco representantes para formar una comisión mixta que debate ambas versiones del proyecto y propone una versión de consenso que luego debe ser sometida a votación en cada Cámara. Si el proyecto propuesto por la comisión mixta es aprobado en ambas Cámaras, se envía al Poder Ejecutivo para su promulgación.

La tabla 1 muestra los estadísticos descriptivos de las variables dependientes e independientes que acabamos de presentar y que utilizamos posteriormente en el análisis descriptivo e inferencial.

Tabla 1. Datos descriptivos de las variables dependientes e independientes de cohesión legislativa de la Cámara de Diputados, Chile, 2006-2014

\begin{tabular}{lccccc}
\hline \multicolumn{1}{c}{ Variables } & $\mathbf{N}$ & Media & Mínimo & Máximo & $\begin{array}{c}\text { Desviación } \\
\text { estándar }\end{array}$ \\
\hline Indicador WUnity Concertación & 5216 & .653 & 0 & .9844 & .222 \\
\hline Indicador WRice Concertación & 5216 & .869 & 0 & 1.000 & .236 \\
\hline Indicador WUnity Alianza & 5216 & .707 & 0 & .9820 & .204 \\
\hline Indicador WRice Alianza & 5216 & .940 & 0 & 1.000 & .161 \\
\hline Indicador WUnity Oficialismo & 5216 & .726 & 0 & .9800 & .187 \\
\hline Indicador WRice Oficialismo & 5216 & .935 & 0 & 1.000 & .171 \\
\hline Indicador WUnity Oposición & 5216 & .634 & 0 & .9800 & .231 \\
\hline Indicador WRice Oposición & 5216 & .874 & 0 & 1.000 & .231 \\
\hline
\end{tabular}




\begin{tabular}{lccccc}
\multicolumn{1}{c}{ Variables } & N & Media & Mínimo & Máximo & $\begin{array}{c}\text { Desviación } \\
\text { estándar }\end{array}$ \\
\hline Dummy cuórum especial & 5216 & 0.217 & 0 & 1 & 0.412 \\
\hline Trámite constitucional & 5148 & 1.5 & 1 & 3 & 0.794 \\
\hline Periodo presidencial Piñera & 5216 & 1.61 & 1 & 2 & 0.487 \\
\hline \% diputados presentes & 5216 & 1 & 0 & 1 & 0.014 \\
\hline Dummy 2008-09 & 5216 & 0 & 0 & 1 & 0.399 \\
\hline Dummy primer año & 5216 & 0.22 & 0 & 1 & 0.414 \\
\hline Dummy último año & 5216 & 0.275 & 0 & 1 & 0.446 \\
\hline
\end{tabular}

Fuente: elaboración propia a partir de datos del Programa de Auditoria de la Democracia, PNUD, para 2006-2009; Campos y Navia (2017) para 2012-2013, y de resultados electorales en el Servicio Electoral y datos propios obtenidos desde la Cámara de Diputados para 2010-2011, disponible en: http://bit.ly/2Z8HAQa.

Desde el retorno de la democracia en 1990, la composición de la Cámara de Diputados, como muestra la tabla 2, ha sido dominada por la centroizquierdista Concertación y la centroderechista Alianza. La Concertación tuvo mayoría de votos y escaños entre 1989 y 2005. En 2009, pese a haber obtenido mayor número de votos, la Concertación recibió un escaño menos que la Alianza. Para el periodo de estudio, la Concertación fue mayoría en la Cámara bajo el Gobierno de Bachelet y la Alianza tuvo una mayoría relativa durante la administración de Piñera.

Tabla 2. Distribución de escaños en elecciones de la Cámara de Diputados, Chile, 1989-2013

\begin{tabular}{ccccc}
\hline \multirow{2}{*}{ Ańo elección } & Concertación $(\mathbf{N})$ & Alianza $(\mathbf{N})$ & Otros $(\mathbf{N})$ & Total \\
\cline { 2 - 5 } & \multicolumn{5}{c}{ \% Votos } \\
\hline 1989 & 51,5 & 34,2 & 14,3 & 100 \\
\hline 1993 & 55,4 & 36,7 & 7,9 & 100 \\
\hline 1997 & 50,5 & 36,3 & 13,2 & 100 \\
\hline 2001 & 47,9 & 44,3 & 7,8 & 100 \\
\hline 2005 & 51,8 & 38,7 & 9,5 & 100 \\
\hline
\end{tabular}




\begin{tabular}{ccccc}
\multirow{2}{*}{ Año elección } & Concertación (N) & Alianza (N) & Otros (N) & Total \\
\cline { 2 - 5 } & \multicolumn{5}{c}{ \% Votos } \\
\hline 2009 & 44,4 & 43,4 & 12,2 & 100 \\
\hline 2013 & 47,7 & 36,2 & 16,1 & 100 \\
\hline 1989 & $57,5(69)$ & $40,00(48)$ & $2,5(3)$ & $100(120)$ \\
\hline 1993 & $58,3(70)$ & $41,7(50)$ & 0 & $100(120)$ \\
\hline 1997 & $57,5(69)$ & $39,2(47)$ & $3,3(4)$ & $100(120)$ \\
\hline 2001 & $51,7(62)$ & $47,5(57)$ & $0,8(1)$ & $100(120)$ \\
\hline $\mathbf{2 0 0 5}$ & $\mathbf{5 4 , 2 ( 6 5 )}$ & $\mathbf{4 5 , 0}(\mathbf{5 4})$ & $\mathbf{0 , 8}(\mathbf{1})$ & $\mathbf{1 0 0}(\mathbf{1 2 0})$ \\
\hline $\mathbf{2 0 0 9}$ & $\mathbf{4 7 , 5 ( 5 7 )}$ & $\mathbf{4 8 , 3}(\mathbf{5 8})$ & $\mathbf{4 , 2}(\mathbf{5})$ & $\mathbf{1 0 0}(\mathbf{1 2 0})$ \\
\hline 2013 & $55,8(67)$ & $40,8(49)$ & $3,3(4)$ & $100(120)$ \\
\hline
\end{tabular}

Fuente: elaboración propia con datos del Servicio Electoral.

Tabla 3. Control del Gobierno y de la mayoría en la Cámara de Diputados en Chile, 2006-2014

\begin{tabular}{lccc}
\hline Año & $\begin{array}{c}\text { Coalición en la Presidencia } \\
\text { de la República }\end{array}$ & $\begin{array}{c}\text { Coalición mayoritaria en } \\
\text { la Cámara de Diputados }\end{array}$ & $\begin{array}{c}\text { \% escańos de la } \\
\text { coalición mayoritaria }\end{array}$ \\
\hline 2006 & Concertación & Concertación & 54,2 \\
\hline 2007 & Concertación & Concertación & 54,2 \\
\hline 2008 & Concertación & Concertación/Alianza & 50,0 \\
\hline 2009 & Concertación & Concertación/Alianza & 50,0 \\
\hline 2010 & Alianza & Alianza & 50,1 \\
\hline 2011 & Alianza & Alianza & 50,1 \\
\hline 2012 & Alianza & Alianza & 50,1 \\
\hline 2013 & Alianza & Alianza & 50,1 \\
\hline
\end{tabular}

Fuente: elaboración propia con datos del Servicio Electoral.

La tabla 3 muestra la coalición que controló la presidencia en cada año y la coalición que controlaba la mayoría en la Cámara de Diputados. Si bien en la elección de 2005 la Concertación ganó mayoría en la Cámara después de que 
cinco diputados del PDC —uno de los partidos de la Concertación — renunciaron a su partido y se sumaron a la Alianza en la votación para la composición de la Mesa de la Cámara en marzo de 2008, el control de la Cámara tuvo que dividirse en partes iguales entre ambas coaliciones en tanto cada coalición tenía el apoyo de sesenta diputados. Después de la elección de diciembre de 2009, la Alianza alcanzó — gracias a un pacto con independientes y diputados de partidos menores - una mayoría suficiente para ejercer el control de la Cámara.

\section{ANÁLISIS DESCRIPTIVO}

Dos de cada tres de las 5216 votaciones de RCV en los ocho años fueron sobre proyectos en primer trámite constitucional, como muestra la tabla 4. En 2010, el primer año del Gobierno de Piñera, solo un 69\% de las votaciones correspondieron a primer trámite. Aunque pudiera tener sentido esperar que las votaciones de primer trámite se concentren en el primer año de Gobierno, en tanto el nuevo presidente introduce sus propias iniciativas legislativas, no hay una relación directa entre el calendario electoral y el porcentaje de votaciones que corresponden al primer trámite constitucional de un proyecto de ley.

Tabla 4. $R C V$ por año y trámite constitucional, 2006-2014 (\% N)

\begin{tabular}{lccccccccc}
\hline \multicolumn{1}{c}{ Trámite } & $\mathbf{2 0 0 6}$ & $\mathbf{2 0 0 7}$ & $\mathbf{2 0 0 8}$ & $\mathbf{2 0 0 9}$ & $\mathbf{2 0 1 0}$ & $\mathbf{2 0 1 1}$ & $\mathbf{2 0 1 2}$ & $\mathbf{2 0 1 3}$ & Total \\
\hline Primero & 78,3 & 69,1 & 80 & 57,6 & 69,6 & 57,4 & 63,8 & 67,3 & $67,5(3.520)$ \\
\hline Segundo & 3,1 & 13,8 & 3,9 & 10,4 & 14,2 & 23,6 & 10,7 & 14,3 & $12,4(648)$ \\
\hline Tercero & 16,3 & 15,6 & 13,8 & 27,3 & 13,9 & 15,3 & 20,3 & 14,7 & $16,7(873)$ \\
\hline $\begin{array}{l}\text { Comisión } \\
\text { Mixta }\end{array}$ & 1,9 & 1,4 & 1,6 & 4,4 & 1,8 & 2,1 & 2,1 & 1,8 & $2,1(107)$ \\
\hline Otras $^{3}$ & 0,4 & 0,2 & 0,7 & 0,2 & 0,6 & 1,6 & 3,1 & 1,9 & $1,3(68)$ \\
\hline Total & 100 & 100 & 100 & 100 & 100 & 100 & 100 & 100 & $100(5.216)$ \\
\hline
\end{tabular}

Fuente: elaboración propia a partir de datos del Programa de Auditoria de la Democracia, PNUD, para 2006-2009, Campos y Navia (2017) para 2012-2013 y datos propios obtenidos desde la Cámara de Diputados para 2010-2011. Disponible en: http://bit.ly/2Z8HAQa.

3 Acuerdo diplomático, reglamento, observaciones del presidente de la República. 
Un 78,3\% de las votaciones correspondió a proyectos de ley de quorum simple, como muestra la tabla 4. De las 1132 votaciones que requieren cuórums especiales, el grueso corresponde a LOC, que requieren mayorías de cuatro séptimos, tres quintos o dos tercios. Con todo, el grueso de las de las votaciones realizadas en el periodo, y en cada año, corresponden a cuestiones que requieren cuórum simple de mayoría de los presentes en sala, como muestra la tabla 5 .

Tabla 5. RCV por año y cuórum, 2006-2014 (\% N)

\begin{tabular}{|c|c|c|c|c|c|c|c|c|c|}
\hline $\begin{array}{l}\text { Tipo de } \\
\text { cuórum }\end{array}$ & 2006 & 2007 & 2008 & 2009 & 2010 & 2011 & 2012 & 2013 & Total \\
\hline Q. simple & 83,3 & 73,3 & 78,4 & 73,4 & 83.0 & 76,7 & 81,1 & 76,1 & $\begin{array}{c}78,3 \\
(4.082) \\
\end{array}$ \\
\hline $\begin{array}{l}\text { Q. } \\
\text { especiales }\end{array}$ & 17,3 & 26,7 & 21,6 & 26,6 & 17,0 & 23,3 & 18,9 & 23,9 & $\begin{array}{c}21,7 \\
(1.132) \\
\end{array}$ \\
\hline \multicolumn{10}{|c|}{ Tipo de cuórum especial } \\
\hline $\begin{array}{l}\text { Q. } \\
\text { calificado }\end{array}$ & 2,3 & 4,4 & 3,4 & 3,9 & 4,8 & 4,6 & 5,8 & 5,9 & $\begin{array}{c}4,6 \\
(241)\end{array}$ \\
\hline LOC & 13 & 18,6 & 14,6 & 17,1 & 10,6 & 17,7 & 11 & 16,2 & $\begin{array}{l}14,7 \\
(766)\end{array}$ \\
\hline $\begin{array}{l}\text { Tres } \\
\text { quintos }\end{array}$ & 0,8 & 0,6 & - & 0,7 & 1,1 & 0,9 & 1,5 & 0,7 & $\begin{array}{l}0,8 \\
(43)\end{array}$ \\
\hline $\begin{array}{l}\text { Dos } \\
\text { tercios }\end{array}$ & 0,6 & 3,2 & 3,6 & 4,9 & 0,6 & 0,1 & 0,6 & 1,1 & $\begin{array}{l}1,6 \\
(82)\end{array}$ \\
\hline $\begin{array}{l}\text { Total } \\
\text { RCV }^{4}\end{array}$ & $\begin{array}{c}100 \\
(484)\end{array}$ & $\begin{array}{c}100 \\
(501)\end{array}$ & $\begin{array}{c}100 \\
(610)\end{array}$ & $\begin{array}{c}100 \\
(432)\end{array}$ & $\begin{array}{c}100 \\
(663)\end{array}$ & $\begin{array}{c}100 \\
(746)\end{array}$ & $\begin{array}{c}100 \\
(827)\end{array}$ & $\begin{array}{l}100 \\
(951)\end{array}$ & $\begin{array}{c}100 \\
(5.214)\end{array}$ \\
\hline
\end{tabular}

Fuente: elaboración propia a partir de datos del Programa de Auditoria de la Democracia, PNUD, para 2006-2009; Campos y Navia (2017) para 2012-2013, y datos propios obtenidos desde la Cámara de Diputados para 2010-2011, disponible en: http://bit.ly/2Z5gJIF.

La figura 1 muestra que el oficialismo está más cohesionado que la oposición en todo el periodo — salvo en 2008 y 2009—, tanto usando WRice como WUnity, pero el oficialismo estuvo más cohesionado en 2010-2014 que

4 Se excluyen dos RCV, uno de 2010 no se codifica el quórum y otro de 2012 que se codifica con cuórum de un tercio. 
en 2006-2010. A su vez, la oposición tuvo menores niveles de unidad en sus votaciones en 2010-2014 que en 2006-2010. Ya que la Alianza fue oposición y Gobierno respectivamente en ambos periodos, podemos concluir que la coalición de derecha estuvo más cohesionada que la Concertación, tanto en calidad de oposición como de Gobierno. Usando el indicador WRice, en 2006-2010 la Concertación tuvo una cohesión de 0,878 y la opositora Alianza de 0,887 . Aunque no es sorpresivo que WUnity tenga valores menores que WRice, la mayor diferencia en WUnity entre el oficialismo y la oposición, que está menos cohesionada, se puede deber a que, especialmente en el primer año, los legisladores de oposición no votan, se abstienen o ausentan más que los del oficialismo. Después, si bien el oficialismo está más cohesionado en sus votaciones que la oposición, la cohesión del oficialismo y de la oposición varía dependiendo de qué coalición esté en el poder.

Figura 1. Cohesión en RCV en Cámara de Diputados de Chile por condición de oficialismo y oposición, 2006-2014

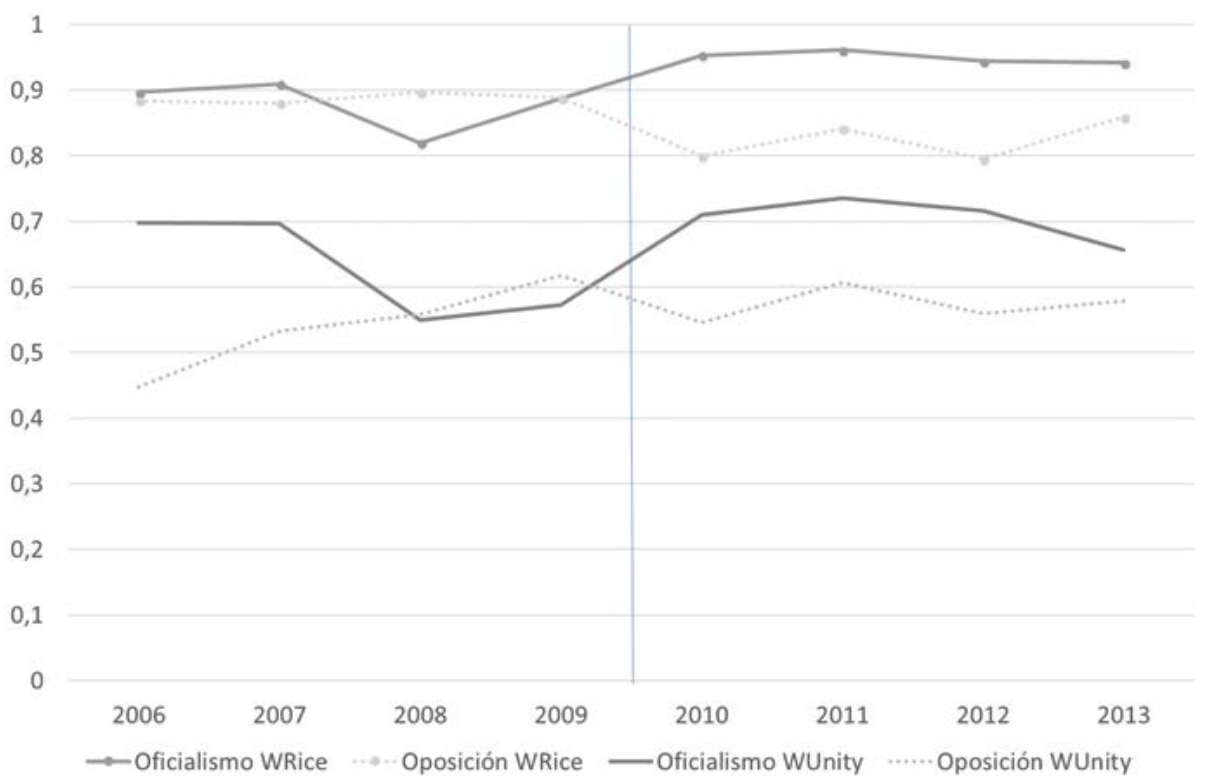

Fuente: elaboración propia a partir de datos del Programa de Auditoria de la Democracia, PNUD, para 2006-2009; Campos y Navia (2017) para 2012-2013, y datos propios obtenidos desde la Cámara de Diputados para 2010-2011, disponible en: http://bit.ly/2Z5gJIF.

La figura 2 muestra la cohesión legislativa por partidos en los dos periodos. En la Alianza casi no hay diferencias entre UDI y RN. Los partidos de la 
Concertación muestran mayor varianza en su cohesión. En parte, esto se puede deber a que hay más partidos en la Concertación que en la Alianza. Cuando hay más partidos en una coalición, es más probable que haya partidos con menos diputados. Un partido con menos diputados, como el PR, en general va a mostrar menos cohesión en tanto la ausencia de un diputado en una votación, en el caso de las votaciones WUnity (o una votación en contra de lo que hace el resto del partido en el caso de WRice) va a tener un impacto mayor sobre la cohesión partidista. Con todo, la evidencia visual indica que los partidos se ordenan en torno a sus coaliciones en sus RCV durante ambos periodos. Luego, parece razonable hacer un análisis más a nivel de coalición que a nivel de partido. En su comportamiento en RCV, los partidos muestran menos diferencias dentro de las coaliciones que entre las coaliciones. Los argumentos de que los partidos en Chile mantienen su independencia y autonomía pudieran ser ciertos en otras dimensiones del quehacer político, pero en la dimensión de la unidad en el comportamiento legislativo los partidos se ordenan en torno a sus coaliciones. No muestran diferencias significativas en su comportamiento dentro de las coaliciones.

Figura 2. Cohesión en RCV en Cámara de Diputados de Chile por partidos por coaliciones, 2006-2014
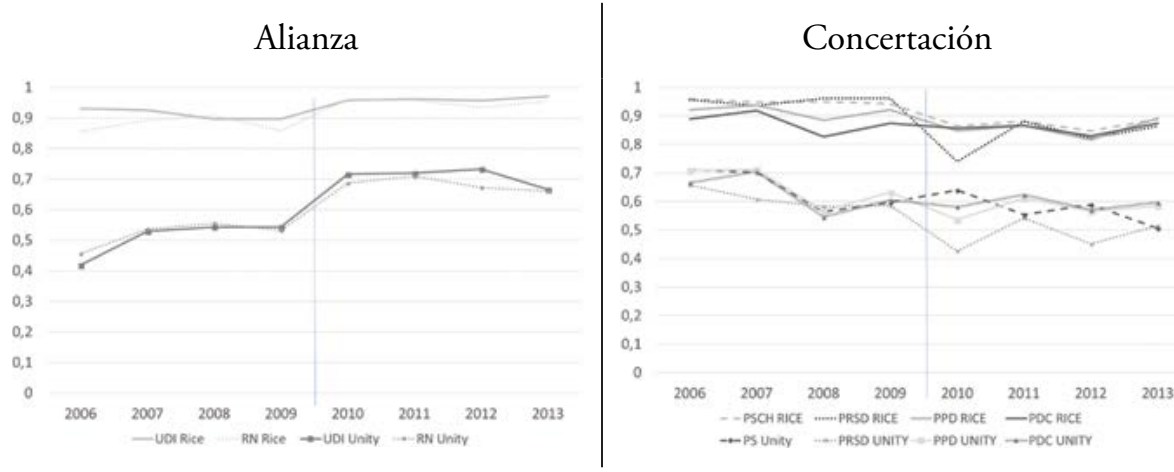

Fuente: elaboración propia a partir de datos del Programa de Auditoria de la Democracia, PNUD, para 2006-2009; Campos y Navia (2017) para 2012-2013, y datos propios obtenidos desde la Cámara de Diputados para 2010-2011, disponible en: http://bit.ly/2Z5gJIF.

Como ya mencionamos, en 2008, el PDC, el principal partido de la Concertación, sufrió una importante escisión. Después de que el senador Adolfo Zaldívar, un histórico líder de la facción más derechista del partido, fue expulsado por discrepancias con el liderazgo, cinco diputados de su 
facción — conocida como Colorines - renunciaron también al partido. En la renovación del liderazgo del Congreso en marzo de 2008, Zaldívar —y Fernando Flores, un senador PPD que también renunció a su partido-, sumaron sus votos a la Alianza y así le dieron control a la oposición de la mesa del Senado. La deserción de los Colorines llevó a un empate de sesenta diputados por coalición, por lo que el control de la Cámara se dividió en partes iguales entre ambas coaliciones. Los Colorines renunciaron también a la bancada PDC y junto a otros independientes crearon una bancada de independientes que, presumiblemente, terminó votando junto a las bancadas de los partidos que conformaban la Alianza.

Para evaluar si eso efectivamente ocurrió, estimamos la cohesión en la votación del PDC con y sin los legisladores que renunciaron. La figura 3 muestra los indicadores WUnity y WRice para los cuatro ańos del periodo para el PDC, distinguiendo entre todos los diputados electos por el PDC y los que se mantuvieron en el PDC después de la renuncia de los díscolos. Aunque renunciaron al PDC, los díscolos siguieron votando de la misma forma en que el resto de los PDC. Luego, parece razonable que, aunque formalmente

Figura 3. Cohesión en RCV en Cámara de Diputados de Chile del PDC, con y sin discolos, 2006-2010

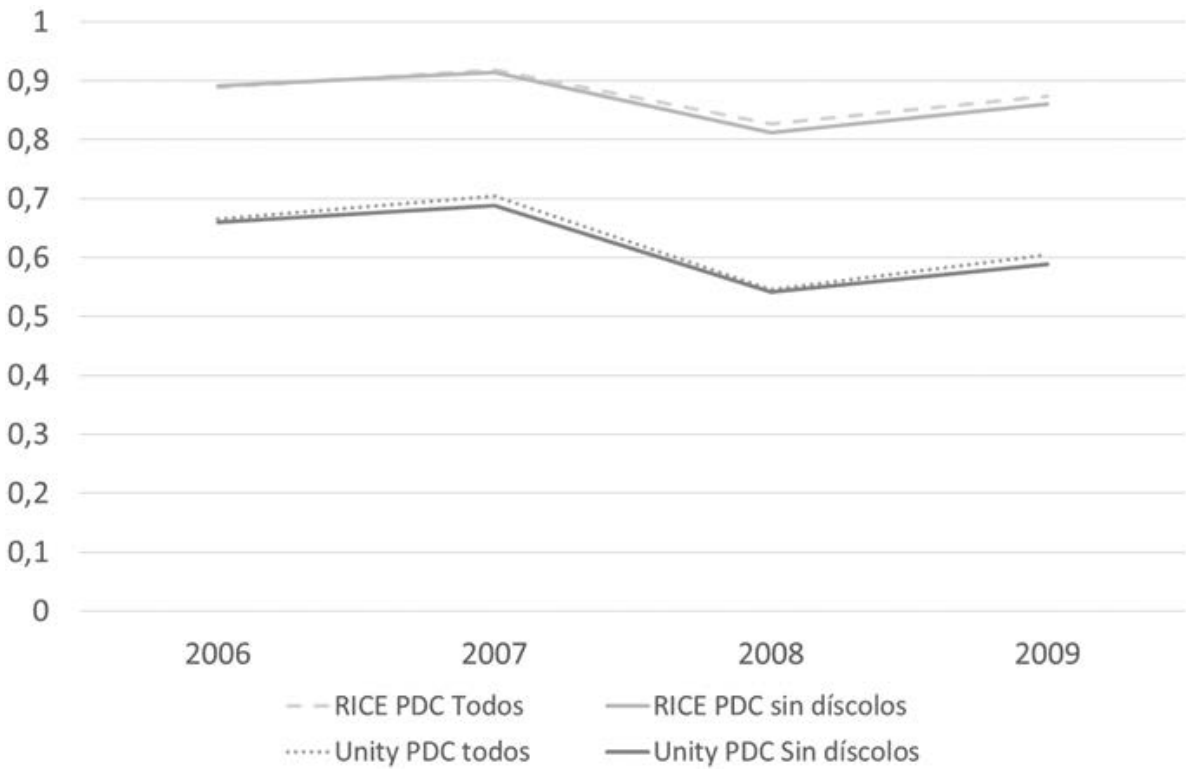

Fuente: elaboración propia a partir de datos del Programa de Auditoria de la Democracia, PNUD, para 2006-2009; Campos y Navia (2017) para 2012-2013, y datos propios obtenidos desde la Cámara de Diputados para 2010-2011, disponible en: http://bit.ly/2Z5gJIF. 
renunciaron al partido y desde 2008 votaron con los diputados de la Alianza en los proyectos de ley más importantes —o al menos así lo reportó la prensa-, los diputados díscolos del PDC se siguieron comportando en sus RCV de forma similar al resto de los diputados PDC que se mantuvieron en el partido.

La figura 4 muestra la cohesión en la votación del oficialismo y la oposición de acuerdo al trámite legislativo. El oficialismo parece estar más cohesionado que la oposición según WUnity y WRice en ambos periodos. Ahora bien, se producen algunas diferencias significativas tanto entre Gobiernos como a medida que avanza el trámite legislativo. En el Gobierno de Bachelet, si bien hay más cohesión siempre en los trámites más avanzados, las

Figura 4. Cohesión en RCV en Cámara de Diputados de Chile por condición de oficialismo y trámite legislativo, 2006-2014
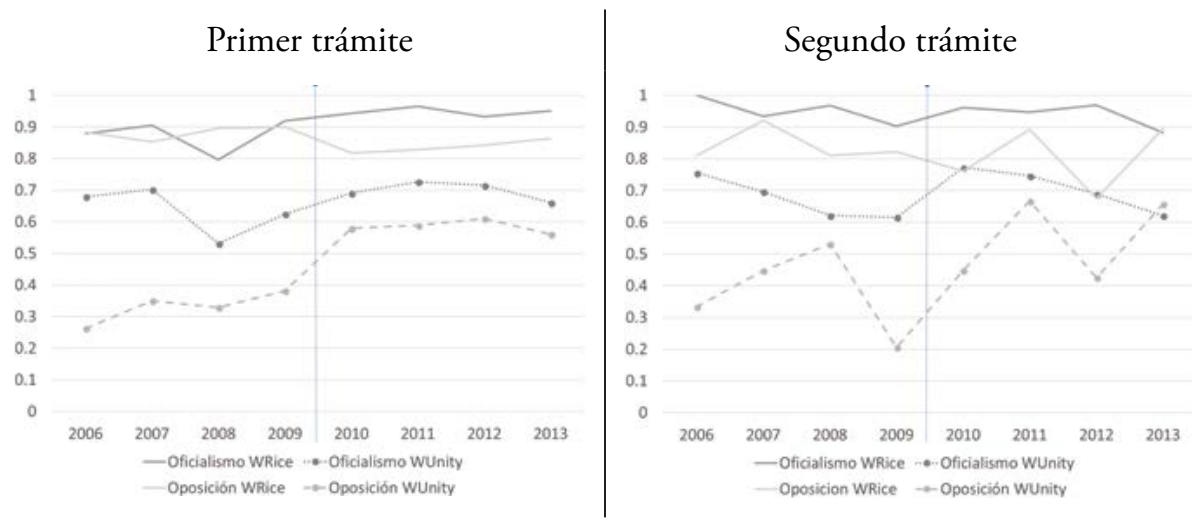

Tercer trámite

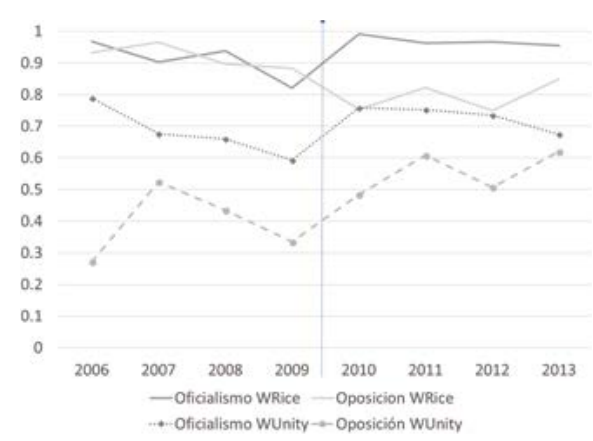

Fuente: elaboración propia a partir de datos del Programa de Auditoria de la Democracia, PNUD, para 2006-2009; Campos y Navia (2017) para 2012-2013, y datos propios obtenidos desde la Cámara de Diputados para 2010-2011, disponible en: http://bit.ly/2Z5gJIF. 
diferencias entre oficialismo y oposición son mayores en WUnity que en WRice. Esto hace suponer que, en vez de votar en contra de la postura mayoritaria de su coalición, los legisladores de oposición parecían preferir ausentarse de la votación. En cambio, en el Gobierno de Piñera, la oposición tuvo menos ausencia en votaciones de los tres trámites. Por eso, en los tres gráficos los valores de las cuatro líneas son más altos en el Gobierno de Piñera que en el de Bachelet. Las diferencias entre los indicadores WRice y WUnity en el Gobierno de Bachelet fueron mayores que en el Gobierno de Piñera.

En general, no obstante, la oposición está menos cohesionada que el oficialismo en todos los trámites legislativos. La mayor diferencia entre oficialismo y oposición se observa en votaciones de tercer trámite constitucional en el Gobierno de Sebastián Piñera, cuando el oficialismo alcanzó una altísima cohesión - más en WRice que en WUnity naturalmente- y la oposición tendió a cohesionarse un poco menos. Si bien hubo años que la oposición se mostró más cohesionada que el oficialismo — como las votaciones de primer trámite en 2008 en WRice-, en general se cumple la expectativa de que el oficialismo se cohesiona más que la oposición, especialmente en votaciones de proyectos que están en etapas más avanzadas.

La figura 5 muestra la cohesión de los diputados por partidos de ambas coaliciones por trámite constitucional. Hay pocas diferencias en la cohesión de los partidos de la Alianza y de la Concertación en votaciones de primer trámite en ambos Gobiernos — con la excepción de la UDI y RN en los dos primeros años del Gobierno de Bachelet, cuando RN mostró menos cohesión que la UDI usando el indicador WRice, lo que puede indicar que los diputados de ese partido prefirieron abstenerse a votar en contra de la posición mayoritaria de ese partido en cuestiones de primer trámite constitucional. Con todo, mientras la unidad en las votaciones de la Alianza aumentó significativamente entre el Gobierno de Bachelet y el de Piñera, la unidad en las votaciones de la Concertación disminuyó solo marginalmente entre dichos Gobiernos.

Si bien se observan algo de menor cohesión en las votaciones de segundo y tercer trámite en los partidos de la Alianza y la Concertación en ambos Gobiernos, pareciera ser que la menor cohesión que se observa entre los partidos de la Concertación responde solo al hecho de que esa coalición está compuesta por un número mayor de partidos que los que componen la Alianza. Ahora bien, es innegable que los partidos de la Concertación mostraron una menor cohesión en el periodo de Piñera, cuando eran oposición, que cuando fueron oficialismo en el periodo de Bachelet. Pero, en general, las dos coaliciones están más cohesionadas en votaciones de segundo y tercer trámite, y cuando son Gobierno que cuando son oposición. No obstante, cuando es Gobierno la Alianza es más disciplinada que la Concertación cuando lo es. Lo mismo ocurre cuando ambas coaliciones son oposición. 
Figura 5. Cohesión en RCV en Cámara de Diputados de Chile por partidos y trámite legislativo, 2006-2014

Alianza

Concertación

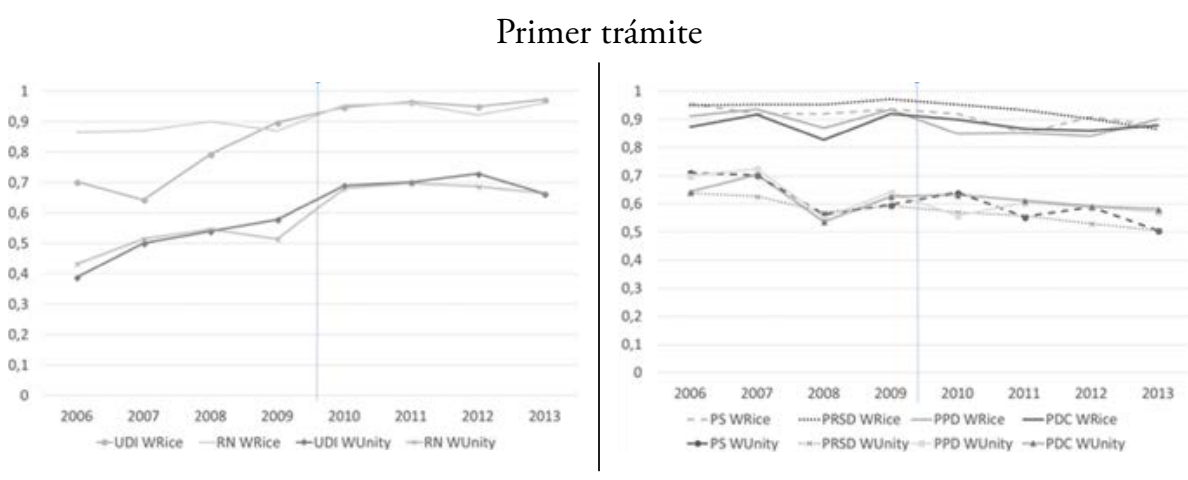

Segundo trámite
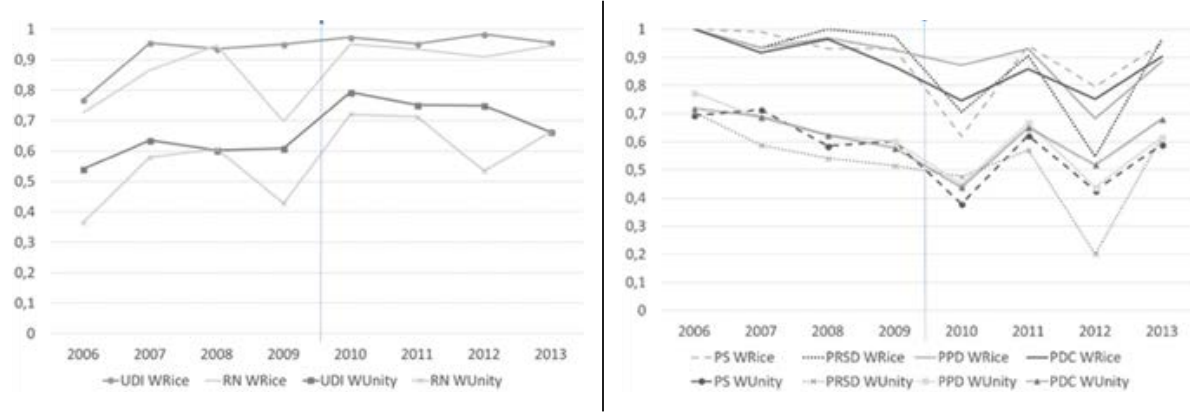

Tercer trámite
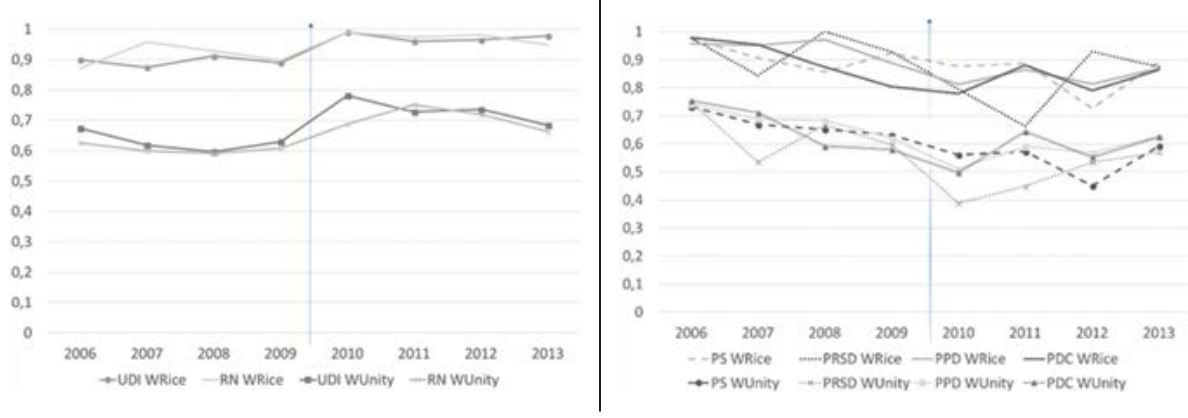

Fuente: elaboración propia a partir de datos del Programa de Auditoria de la Democracia, PNUD, para 2006-2009; Campos y Navia (2017) para 2012-2013, y datos propios obtenidos desde la Cámara de Diputados para 2010-2011, disponible en: http://bit.ly/2Z5gJIF. 
La figura 6 muestra la cohesión en ambos Gobiernos según el cuórum requerido en cada proyecto de ley que se votaba. En general, el oficialismo está más cohesionado que la oposición. No hay grandes diferencias entre las votaciones que requerían cuórum simple y aquellas que requerían cuórums especiales. Ahora bien, en 2008, cuando la Concertación experimentó el quiebre con la renuncia de los diputados PDC, la cohesión de esa coalición se vio afectada en votaciones de cuórum simple, pero no en votaciones de cuórums especiales, lo que lleva a pensar que el conflicto dentro del PDC no llegó a producir quiebras en las posiciones de los legisladores respecto a votaciones que eran consideradas de más importancia en el trámite legislativo. A su vez, en el Gobierno de Piñera la oposición pareció demostrar menos cohesión que la que mostró la oposición en el Gobierno de Bachelet en ambos indicadores de cohesión. Pero en el último año del Gobierno de Piñera, mientras el oficialismo se mostró menos cohesionado, la oposición mostró mayor cohesión.

Figura 6. Cohesión en RCV en Cámara de Diputados de Chile por condición de oficialismo y tipo de cuórum, 2006-2014
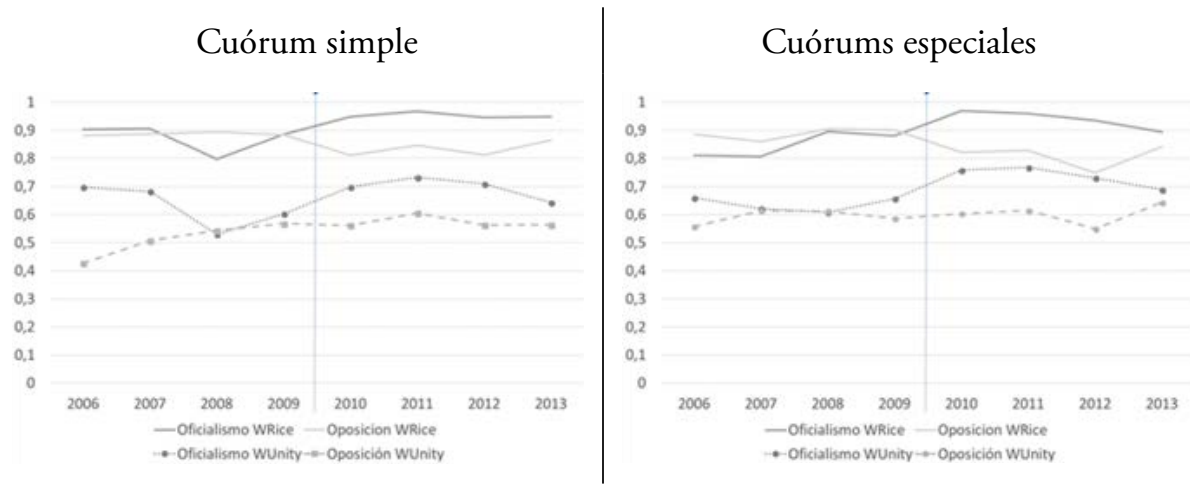

Fuente: elaboración propia a partir de datos del Programa de Auditoria de la Democracia, PNUD, para 2006-2009; Campos y Navia (2017) para 2012-2013, y datos propios obtenidos desde la Cámara de Diputados para 2010-2011, disponible en: http://bit.ly/2Z5gJIF.

La figura 7 muestra la cohesión en los partidos de cada coalición en ambos periodos, de acuerdo al cuórum de la votación de cada RCV. Nuevamente, no hay diferencias significativas entre los partidos de cada coalición. Esto confirma nuestra propuesta de analizar a los partidos como parte de una coalición más que como partidos de forma individual. Si bien hay algo menos de cohesión en los partidos de la Concertación que en los de la Alianza, el hecho que la Concertación viera su contingente dividido entre cuatro partidos (y cinco a partir de 2010), mientras que el contingente legislativo de la Alianza se divide solo entre 
Figura 7. Cohesión en RCV en Cámara de Diputados de Chile por partidos y tipo de cuórum, 2006-2014

Alianza

Concertación

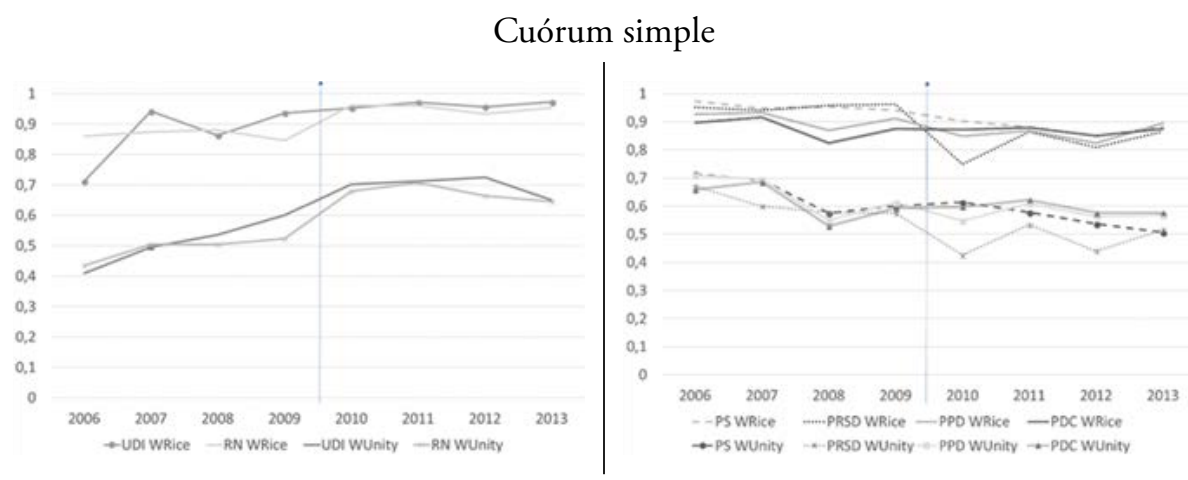

Cuórums especiales
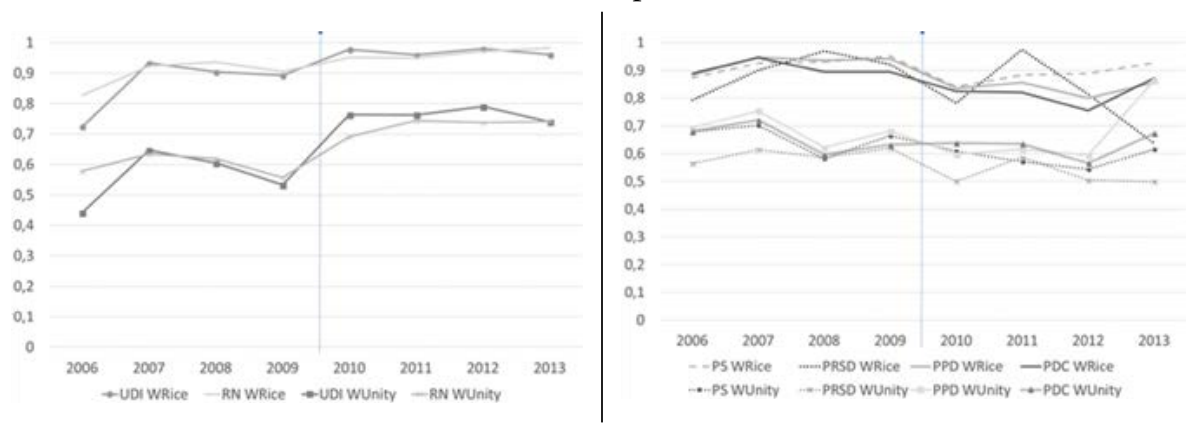

Fuente: elaboración propia a partir de datos del Programa de Auditoria de la Democracia, PNUD, para 2006-2009; Campos y Navia (2017) para 2012-2013, y datos propios obtenidos desde la Cámara de Diputados para 2010-2011, disponible en: http://bit.ly/2Z5gJIF.

dos partidos explica esa mayor varianza. Con todo, mientras que los partidos de la Alianza se mostraron más cohesionados durante el Gobierno de Piñera, los partidos de la Concertación mostraron más cohesión en el Gobierno de Bachelet, lo que confirma la observación anterior de que los partidos se cohesionan más en sus RCV cuando son oficialismo que cuando son oposición.

\section{ANÁLISIS INFERENCIAL}

Para evaluar el efecto combinado de las distintas variables y testar nuestras dos hipótesis, realizamos un análisis inferencial con ocho modelos de 
regresión de mínimos cuadrados ordinarios (MCO). La variable dependiente es la cohesión en la votación de las coaliciones de acuerdo a los indicadores WRice y WUnity. Las variables independientes de interés son una dummy que distingue al periodo presidencial de Pińera, para diferenciar la condición de oficialismo y oposición de las dos coaliciones, y dos indicadores para distinguir la importancia de una votación: una dummy que distingue las votaciones sobre proyectos que requieren cuórum especial y una variable que identifica el trámite legislativo (primero, segundo o tercer trámite). Adicionalmente, como variables de control incluimos el porcentaje de diputados presentes, una dummy que identifica el periodo 2008-2009 (cuando cinco diputados del PDC renunciaron y se sumaron a la Alianza), y dummies para las votaciones que se realizaron el primer año de gobierno (2006 y 2010), y el último año de gobierno (2009 y 2013). Finalmente, también incluimos como variable de control el indicador WUnity o WRice para la coalición opuesta de la que se analiza en cada modelo. Así, para el modelo 1, que explica los determinantes de la cohesión en la Concertación, la variable Cohesión diputados coalición opuesta incluye el valor WUnity para la Alianza.

Los modelos de la tabla 6 muestran un efecto significativo del cuórum sobre la cohesión en la votación de ambas coaliciones, pero solo con el indicador WUnity. Mientras mayor es el cuórum, mayor es la cohesión. Pero como esto no ocurre con el indicador WRice - que no considera las ausencias como votos en contra de la mayoría de los diputados de la coalición- podemos inferir que la importancia del cuórum hace que, cuando un diputado no quiere votar contra la mayoría de su coalición, simplemente se ausenta de la votación. Por eso, el efecto de la variable cuórum es significativa solo con el indicador WUnity.

El efecto de la variable trámite constitucional es significativo solo para la cohesión de la Alianza, tanto usando WUnity como WRice. En la Concertación, la votación de un proyecto en trámite más avanzado no afecta la cohesión entre los diputados de la coalición. Si bien la Alianza se cohesionó más en las votaciones de trámites más avanzados, esa coalición mostraba menos cohesión en las votaciones de primer trámite. En cambio, la Concertación en general mostró similar cohesión en todas las votaciones, independientemente del trámite.

Comprensiblemente, el efecto de la dummy Gobierno de Piñera es positivo para la cohesión de la Alianza y negativo para la Concertación.

La cantidad de diputados presentes en la votación no tiene efectos sobre la unidad de cada coalición. Como no debiera sorprender, el efecto de la dummy 2008-2009 es negativo sobre la cohesión en la votación de la Concertación. Ya que consideramos como parte de la Concertación a todos los diputados electos en esa coalición, cuando algunos de esos diputados la 
abandonaron y se unieron a la Alianza, se observa una menor cohesión en la votación de la Concertación. Sin embargo, para nuestra sorpresa, la cohesión en la Alianza también se vio negativamente afectada. Ya que entonces se hablaba de que había un número creciente de diputados díscolos - que disminuyen la cohesión respecto a las posiciones de sus coaliciones- pudiéramos pensar que esa condición de díscolo se extendió también a algunos diputados de la derechista Alianza. Esto es, la cohesión disminuyó en ambas coaliciones tanto porque algunos diputados renunciaron a sus partidos como porque otros simplemente dejaron de votar a la par con el resto de los legisladores de su coalición, aunque no hayan renunciado a sus partidos.

Sorpresivamente, el efecto de la variable último año es distinto para la Alianza y la Concertación. Mientras la Concertación muestra mayor cohesión en las votaciones de último año cuando se usa el indicador WRice, la Alianza está siempre menos cohesionada en el último año, tanto cuando se usa WRice como WUnity.

Finalmente, el efecto de la cohesión en la coalición opuesta es positivo sobre la cohesión en la votación de ambas coaliciones. Si la Concertación vota más unidamente, la Alianza también lo hará. Esto refleja que ambas coaliciones tienden a dar igual importancia a los distintos tipos de votaciones. A diferencia de lugares en que algunos temas son prioritarios para un partido y los otros partidos pudieran no mostrar mayor interés, en el caso de Chile cuando una coalición se ordena en torno a una discusión legislativa, la otra coalición también se ordena en torno a esa votación, ya sea en oposición o para apoyarla.

Respecto a las dos hipótesis que planteamos, hay evidencia para sostener parcialmente la validez de la primera hipótesis. El oficialismo está más cohesionado que la oposición - salvo en votaciones de tercer tramite constitucional, cuando ambas coaliciones tienden a cohesionarse más-. Ahora bien, hay diferencias en la cohesión de la Alianza y la Concertación cuando cada coalición es oficialismo. Tanto en su condición de oficialismo como de oposición, la Alianza estuvo más cohesionada que la Concertación.

También encontramos evidencia a favor de la segunda hipótesis. Ambas coaliciones se cohesionan más cuando el cuórum es más alto y el trámite legislativo más avanzado, aunque como hay menor cantidad de votaciones con cuórums más altos y trámites más avanzados, también vemos varianza por años en la cohesión de ambas coaliciones en este tipo de votaciones más importantes. Pero al agregar todas las votaciones de todos los años, ambas coaliciones - Alianza y Concertación, oficialismo y oposición — son más cohesionadas cuando el cuórum es más alto y el trámite más avanzado. Esto es, cuando las votaciones importan más, las coaliciones se muestran más unidas en sus votaciones. 
Tabla 6. Modelos de regresión lineal sobre cohesión legislativa en la Cámara de Diputados de Chile, 2006-20145

\begin{tabular}{|c|c|c|c|c|c|c|c|c|}
\hline Variables & $\begin{array}{l}\text { Modelo } 1 \\
\text { WUnity } \\
\text { Concertac }\end{array}$ & $\begin{array}{l}\text { Modelo } 2 \\
\text { WRice } \\
\text { Concertac }\end{array}$ & $\begin{array}{c}\text { Modelo } 3 \\
\text { WUnity } \\
\text { Alianza }\end{array}$ & $\begin{array}{c}\text { Modelo } 4 \\
\text { WRice } \\
\text { Alianza }\end{array}$ & $\begin{array}{c}\text { Modelo } 5 \\
\text { WUnity } \\
\text { Oficialismo }\end{array}$ & $\begin{array}{c}\text { Modelo } 6 \\
\text { WRice } \\
\text { Oficialismo }\end{array}$ & $\begin{array}{c}\text { Modelo } 7 \\
\text { WUnity } \\
\text { Oposición }\end{array}$ & $\begin{array}{c}\text { Modelo } 8 \\
\text { WRice } \\
\text { Oposición }\end{array}$ \\
\hline Cuórum & $\begin{array}{c}, 023^{* *} \\
(, 007)\end{array}$ & $\begin{array}{l}-, 019^{*} \\
(, 008)\end{array}$ & $\begin{array}{l}-, 008 \\
(, 005)\end{array}$ & $\begin{array}{l}-, 010 \\
(, 005)\end{array}$ & $\begin{array}{l}, 006 \\
(, 006)\end{array}$ & $\begin{array}{c}, 005 \\
(, 006)\end{array}$ & $\begin{array}{c}, 001 \\
(, 008)\end{array}$ & $\begin{array}{c}, 004 \\
(, 008)\end{array}$ \\
\hline Trámite & $\begin{array}{l}-, 013^{*} \\
(, 006)\end{array}$ & $\begin{array}{l}-, 008 \\
(, 004)\end{array}$ & $\begin{array}{l}, 001 \\
(, 002)\end{array}$ & $\begin{array}{l}, 006^{*} \\
(, 003)\end{array}$ & $\begin{array}{l}, 000 \\
(, 003)\end{array}$ & $\begin{array}{l}, 000 \\
(, 003)\end{array}$ & $\begin{array}{l}, 003 \\
(, 004)\end{array}$ & $\begin{array}{l}, 005 \\
(, 004)\end{array}$ \\
\hline $\begin{array}{l}\text { Periodo } \\
\text { presid } \\
\text { Piñera }\end{array}$ & $\begin{array}{c}-, 089^{* *} \\
(, 007)\end{array}$ & $\begin{array}{c}-, 078^{* *} \\
(, 009)\end{array}$ & $\begin{array}{c}, 070^{* *} \\
(, 006)\end{array}$ & $\begin{array}{l}, 054^{* *} \\
(, 006)\end{array}$ & $\begin{array}{l}-, 012 \\
(, 007)\end{array}$ & $\begin{array}{c}, 004 \\
(, 007)\end{array}$ & $\begin{array}{l}, 002 \\
(, 009)\end{array}$ & $\begin{array}{l}, 007 \\
(, 009)\end{array}$ \\
\hline $\begin{array}{l}\% \\
\text { diputados } \\
\text { presentes }\end{array}$ & $\begin{array}{c}-, 012^{* *} \\
(, 000)\end{array}$ & $\begin{array}{c}, 002^{* *} \\
(, 000)\end{array}$ & $\begin{array}{c}-, 012^{* *} \\
(, 000)\end{array}$ & $\begin{array}{c}-, 001^{* *} \\
(, 000)\end{array}$ & $\begin{array}{l}-, 000 \\
(, 000)\end{array}$ & $\begin{array}{c}-, 001^{* *} \\
(, 000)\end{array}$ & $\begin{array}{l}, 000 \\
(, 000)\end{array}$ & $\begin{array}{l}, 001^{* *} \\
(, 000)\end{array}$ \\
\hline $\begin{array}{l}\text { Dummy } \\
2008-09\end{array}$ & $\begin{array}{l}-, 043^{*} \\
(, 010)\end{array}$ & $\begin{array}{c}-, 038^{* *} \\
(, 012)\end{array}$ & $\begin{array}{c}-, 036^{* *} \\
(, 007)\end{array}$ & $\begin{array}{l}, 012 \\
(, 008)\end{array}$ & $\begin{array}{l}-, 012 \\
(, 010)\end{array}$ & $\begin{array}{l}-, 007 \\
(, 009)\end{array}$ & $\begin{array}{l}, 019 \\
(, 012)\end{array}$ & $\begin{array}{l}, 003 \\
(, 012)\end{array}$ \\
\hline $\begin{array}{l}\text { Dummy } \\
\text { primer } \\
\text { año }\end{array}$ & $\begin{array}{l}-, 013^{*} \\
(, 007)\end{array}$ & $\begin{array}{c}-, 003^{* *} \\
(, 008)\end{array}$ & $\begin{array}{l}-, 011^{*} \\
(, 005)\end{array}$ & $\begin{array}{c}, 004 \\
(, 006)\end{array}$ & $\begin{array}{l}, 010 \\
(, 007)\end{array}$ & $\begin{array}{c}, 002 \\
(, 006)\end{array}$ & $\begin{array}{c}, 004 \\
(, 008)\end{array}$ & $\begin{array}{c}, 022^{* *} \\
(, 008)\end{array}$ \\
\hline $\begin{array}{l}\text { Dummy } \\
\text { último año }\end{array}$ & $\begin{array}{c}, 030^{* *} \\
(, 006)\end{array}$ & $\begin{array}{c}, 036^{* *} \\
(, 008)\end{array}$ & $\begin{array}{c}-, 018^{* *} \\
(, 005)\end{array}$ & $\begin{array}{l}-, 012^{*} \\
(, 005)\end{array}$ & $\begin{array}{c}-, 014^{*} \\
(, 007)\end{array}$ & $\begin{array}{c}-, 012^{*} \\
(, 006)\end{array}$ & $\begin{array}{l}, 021^{* *} \\
(, 008)\end{array}$ & $\begin{array}{c}, 003 \\
(, 008)\end{array}$ \\
\hline $\begin{array}{l}\text { Cohesión } \\
\text { coalición } \\
\text { opuesta }\end{array}$ & $\begin{array}{c}-, 346^{* *} \\
(, 018)\end{array}$ & $\begin{array}{c}, 092^{* *} \\
(, 020)\end{array}$ & $\begin{array}{c}-, 196^{* *} \\
(, 010)\end{array}$ & $\begin{array}{l}, 043^{* *} \\
(, 009)\end{array}$ & $\begin{array}{l}103^{* *} \\
(, 011)\end{array}$ & $\begin{array}{c}, 031^{* *} \\
(, 010)\end{array}$ & $\begin{array}{l}, 158^{* *} \\
(, 017)\end{array}$ & $\begin{array}{l}, 058^{* *} \\
(, 019)\end{array}$ \\
\hline Constante & $\begin{array}{l}, 151 \\
(, 022)\end{array}$ & $\begin{array}{c}, 810 \\
(, 032)\end{array}$ & $\begin{array}{l}, 197 \\
(, 017)\end{array}$ & $\begin{array}{l}, 741 \\
(, 020)\end{array}$ & $\begin{array}{c}, 692 \\
(, 025)\end{array}$ & $\begin{array}{l}1,007 \\
(, 022)\end{array}$ & $\begin{array}{l}, 463 \\
(, 032)\end{array}$ & $\begin{array}{c}, 717 \\
(, 033)\end{array}$ \\
\hline $\begin{array}{l}\text { R2- } \\
\text { ajustado }\end{array}$ & ,295 & ,027 & ,530 & ,033 & ,017 & ,009 & ,017 & ,006 \\
\hline $\mathbf{N}$ & 5.145 & 5.145 & 5.145 & 5.145 & 5.145 & 5.145 & 5.145 & 5.145 \\
\hline
\end{tabular}

* Significativo al $0.05 .{ }^{* *}$ Significativo al 0.01 .

Fuente: elaboración propia a partir de datos del Programa de Auditoria de la Democracia, PNUD, para 2006-2009; Campos y Navia (2017) para 2012-2013, y datos propios obtenidos desde la Cámara de Diputados para 2010-2011, disponible en: http://bit.ly/2Z5gJIF.

5 Si bien hubo 5216 roll call votes, hay 71 casos en los que no se indica a qué trámite constitucional correspondió la votación. De ahí que el $\mathrm{N}$ para la regresión sea de 5145 . 
Por cierto, no hay evidencia de que la Alianza estuviera más cohesionada en el primer año del Gobierno de Piñera que la Concertación en el primer año del Gobierno de Bachelet. Después, el hecho de que la Alianza haya sido oficialismo por primera vez desde el retorno de la democracia no hizo que esa coalición mostrara más unidad en su comportamiento que lo que mostró la Concertación en el primer año del Gobierno de Bachelet, que fue el cuarto Gobierno consecutivo en que la coalición de centroizquierda estuvo en el poder.

A su vez, la Concertación estuvo más cohesionada en el último año de Bachelet que la Alianza en el último año de Piñera. De hecho, la Alianza estuvo menos cohesionada en el último año en el Gobierno de Piñera que en años anteriores. La Concertación tuvo más unidad partidaria en el último año de Bachelet que en ańos intermedios y menos cohesionada en el primer año que en ańos intermedios. Esto puede relacionarse tanto con las dinámicas que explicaron el proceso político en el último año de cada Gobierno - con Bachelet, que dejó el poder con altos niveles de aprobación, y Piñera que, si bien mejoró su aprobación en el último año, dejó el poder con aprobación bastante menor a la de Bachelet-. Aunque también esto pudiera deberse a consideraciones prospectivas por parte de los legisladores. Mientras las elecciones de 2009 fueron muy reñidas entre los candidatos de la Alianza y la Concertación - lo que pudo inducir a mayor cohesión en el oficialismo ese año-, la enorme ventaja que tuvo Bachelet en la campańa electoral de 2013 pudo haber inducido a una mayor indisciplina en el oficialismo de derecha, en tanto ese sector pareció tener pocas probabilidades de mantenerse en el poder después del fin del Gobierno de Piñera.

\section{CONCLUSIÓN}

El comportamiento de los partidos en el poder legislativo se explica por variables institucionales y por cuestiones culturales. En tanto el diseño institucional no varíe, los cambios en el comportamiento legislativo de los partidos se pueden explicar por cuestiones culturales, especialmente cuando la coalición en el poder cambia de color político. Bajo las mismas reglas, distintos partidos se pueden comportar de forma distinta cuando son Gobierno y cuando son oposición.

Desde el retorno de la democracia en 1990, el sistema político chileno ha visto una cerrada competencia entre dos grandes coaliciones, la centroizquierdista Concertación, que gobernó entre 1990 y 2010, y la centroderechista Alianza, que gobernó entre 2010 y 2014. Ambas coaliciones tuvieron mayoría 
en la Cámara de Diputados en los periodos que fueron Gobierno. Si bien la alternancia en el poder es una característica propia de la democracia, las dinámicas de comportamiento político que se producen en una democracia estable cuando cambia la coalición en el poder pudieran también ser diferentes. Con datos de 5216 RCV — votaciones en sala — de la Cámara de Diputados entre 2006 y 2014, evaluamos el comportamiento de las coaliciones en la forma en que votaron proyectos de ley.

Primero, confirmamos que el comportamiento de los legisladores puede ser analizado en tanto su pertenencia a las coaliciones más que a los partidos. Esto porque existe poca varianza en la cohesión de la votación de los distintos partidos que componen cada coalición. Luego, más que analizar las diferencias entre los partidos miembros de una coalición — cuestión que se ve influenciada por el número de partidos que componen cada coalición - resulta conveniente evaluar la cohesión en las coaliciones.

Reportamos que, en general, la coalición de Gobierno está más cohesionada que la de oposición. Aunque ambas coaliciones se cohesionan más cuando se trata de votaciones más importantes — ya sea porque requieren de mayor cuórum para ser aprobadas o porque están en trámites legislativos más avanzados- la coalición oficialista se cohesiona más que la de oposición. A su vez, reportamos que, si bien hay diferencias en la cohesión de las coaliciones dependiendo del calendario legislativo, la Concertación en general tiende a cohesionarse más como coalición de Gobierno hacia el final del periodo mientras que la Alianza muestra menor cohesión hacia el final del periodo cuando fue Gobierno.

Si bien hay patrones comunes al comportamiento de las dos coaliciones multipartidistas en Chile tanto cuando son Gobierno como cuando son oposición, también hay diferencias en la forma en que ambas coaliciones ejercen su papel como coalición oficialista y como coalición de oposición. En general, la Alianza se cohesiona más que la Concertación, tanto en su condición de oficialismo como cuando es oposición, pero la Concertación parece ser más capaz de unirse en temas que considera importantes y relevantes —en aquellas que requieren cuórum más alto y las que están más avanzadas en el proceso legislativo-.

El caso de Chile confirma que, si bien el diseño institucional incide en el comportamiento de los legisladores en el Congreso, hay cuestiones de cultura partidista que también afecta el nivel de cohesión de los partidos en el Congreso cuando son Gobierno y cuando son oposición. Los estudios sobre el comportamiento legislativo de los partidos debieran tomar en cuenta tanto las variables de diseño institucional como las dinámicas de cultura partidista para dar mejores respuestas sobre por qué los partidos se comportan de la forma como lo hacen en sus votaciones en el Congreso. 


\section{Bibliografía}

Alemán, E. (2006). Policy Gatekeepers in Latin American Legislatures. Latin American Politics and Society, 48 (3), 98-120. Disponible en: https://doi.org/10.1353/lap.2006.0028.

— y Calvo, E. (2008). Analyzing Legislative Success in Latin America: The Case of Democratic Argentina. En G. O’Donnell, J. Tulchin y A. Varas (eds.). New Voices in the Study of Democracy in Latin America (pp. 7-37). Washington D. C.: Woodrow Wilson International Center for Scholars.

- y Navia, P. (2009). Institutions and the Legislative Success of 'Strong' Presidents: An Analysis of Government Bills in Chile. Journal of Legislative Studies, 15 (4), 401-419. Disponible en: https://doi.org/10.1080/13572330903302471.

— y Saiegh S. M. (2007). Legislative preferences, political parties, and coalition unity in Chile. Comparative Politics, 39 (3), 253-272. Disponible en: https://www.jstor.org/stable/20434040.

Aninat, C. (2006). El proceso legislativo chileno: Análisis del proyecto de ley de rentas vitalicias. Santiago de Chile: Departamento de Estudios de Superintendencia de Administradoras de Fondos de Pensiones de Chile.

— , Londregan, J., Navia, P. y Vial, J. (2006). Political Institutions, Policymaking Processes, and Policy Outcomes in Chile. Washington D.C.: Inter-American Development Bank.

_ , Londregan, J., Navia, P. y Vial, J. (2008). Political Institutions, Policymaking Processes, and Policy Outcomes in Chile. En E. Stein y M. Tommasi (eds.). Policy Making in Latin America. How Politics Shapes Policies (pp.155-198). Cambridge: David Rockefeller Center for Latin American Studies.

Ansolabehere, S. y Edward, P. (2010). Constituents' Responses to Congressional Roll-Call Voting. American Journal of Political Science, 54 (3), 583-597. Disponible en: https:// doi.org/10.1111/j.1540-5907.2010.00448.x.

Baldez, L. y Carey, J. (1999). Presidential agenda control and spending policy: Lessons from general Pinochet's constitution. American Journal of Political Science, 43 (1), 29-55. Disponible en: https://doi.org/10.2307/2991784.

Calvo, E. (2007). The Responsive Legislature: Public Opinion and Law Making in a Highly Disciplined Legislature. British Journal of Political Science, 37 (2), 263-280. Disponible en: https://doi.org/10.1017/S0007123407000130.

Campos-Parra, H. y Navia, P. (2017). Disciplina legislativa en la Cámara de Diputados de Chile, 2010-2014. Politica y Gobierno, 24 (1), 81-123.

Carey, J. (2002). Parties, Coalitions, and the Chilean Congress in the 1990s. En S. Morgerstern y B. Nacif (eds.). Legislative Politics in Latin America. New York: Cambridge University Press.

- (2006). Legislative organization. En R.A.W. Rhodes, S. Binder y B. Rockman (eds.). The Oxford Handbook of Political Institutions. New York: Oxford University Press.

- (2007). Competing principals, political institutions, and party unity in legislative voting. American Journal of Political Science, 51 (1), 92-107. Disponible en: https://doi. org/10.1111/j.1540-5907.2007.00239.x. 
- (2009). Legislative Voting and Accountability. New York: Cambridge University Press. Disponible en: https://bit.ly/2Yw8zZG.

Clausen, A. (1967). The measurement of legislative group behavior. Midwest Journal of Political Science, 11 (2), 212-224. Disponible en: https://doi.org/10.2307/2109112.

Close, C. (2018). Parliamentary party loyalty and party family: The missing link? Party Politics, 24 (2), 209-219. Disponible en: https://doi.org/10.1177/1354068816655562.

Colomer, J. (2005). It's parties that choose electoral systems (or, Duverger's laws upside down). Political Studies, 53 (1), 1-21. Disponible en: https://doi.org/10.1111/j.14679248.2005.00514.x.

Covington, C., Wrighton, M. y Kinney, R. (1995). A 'Presidency-Augmented' Model of Presidential Success in Roll Call Votes. American Journal of Political Science, 39 (4), 10011024. Disponible en: https://doi.org/10.2307/2111667.

Cox, G. (1997). Making votes count. Strategic Coordination in the World's Electoral Systems. Cambridge: Cambridge University Press. Disponible en: https://doi.org/10.1017/ CBO9781139174954.

- (2006). The Organization of Democratic Legislatures. En B. Weingast y D. Wittman (eds.). The Oxford Handbook of Political Economy (pp. 141-161). New York: Oxford University Press.

- y McCubbins, M. (2005). Setting the agenda: Responsible party government in the US House of Representatives. Cambridge, US.: Cambridge University Press. Disponible en: https://doi.org/10.1017/CBO9780511791123.

- y McCubbins, M. (2007). Legislative leviathan: Party government in the House: Cambridge, US.: Cambridge University Press. Disponible en: https://doi.org/10.1017/ CBO9780511810060.

Duverger, M. (1954). Political Parties. New York: Wiley.

Figueiredo, A. y Limongi, F. (2000). Presidential Power, Legislative Organization, and Party Behavior in Brazil. Comparative Politics, 32 (2), 151-170. Disponible en: https://doi. org/10.2307/422395.

Golder, S. (2006). Logic of Pre-electoral coalition formation (Parliaments and Legislatures). Columbus: Ohio State University Press. Disponible en: https://doi.org/10.1017/ S0007123406000123.

Grofman, B. y Lijphart, A. (eds.) (1986). Electoral Laws and Their Political Consequences. New York: Agathon Press.

Grumm, J. (1963). A factor analysis of legislative behavior. Midwest Journal of Political Science, 7 (4), 336-356. Disponible en: https://doi.org/10.2307/2108806.

Lijphart, A. (1990). The political consequences of electoral laws: 1945-1985. American Political Science Review, 84, 481-496. Disponible en: https://doi.org/10.2307/1963530.

Linz, J. y Valenzuela, A. (1994). The Failure of Presidential Democracy. The Case of Latin America. (vol. 2). Baltimore: John Hopkins University.

- (1998). Las crisis del presidencialismo. Madrid: Alianza Editorial.

Lipset, S. y Rokkan, S. (eds.) (1967). Party Systems and Voter Alignments: Cross-National Perspectives. New York: Free Press.

Londregan, J. (2000). Legislative Institutions and Ideology in Chile. New York: Cambridge University Press. Disponible en: https://doi.org/10.1017/CBO9780511571565. 
Morgenstern, S. (2004). Patterns of Legislative Politics. Roll-Call Voting in Latin America and the United States. New York: Cambridge University Press. Disponible en: https://doi. org/10.1017/CBO9780511510328.

— y Nacif, B. (eds.) (2002). Legislative Politics in Latin America. New York: Cambridge University Press. Disponible en: https://doi.org/10.1017/CBO9780511615665.

Özbudun, E. (1970). Party cohesion in Western democracies: A causal analysis: Beverly Hills: Sage.

Poole, K. (2005). Spatial Models of Parliamentary Voting. New York: Cambridge University Press. Disponible en: https://doi.org/10.1017/CBO9780511614644.

- y Rosenthal, H. (2000). Congress: A political-economic history of roll call voting. New York: Oxford University Press on Demand.

Rice, S. (1925). The behavior of legislative groups: a method of measurement. Political Science Quarterly, 40 (1), 60-72. Disponible en: https://doi.org/10.2307/2142407.

Sartori, G. (1986). The Influence of Electoral Systems: Faulty Laws or Faulty Method? En B. Grofman y A. Lijphart (eds.). Electoral Laws and Their Political Consequences (pp. 43-48). New York: Agathon Press.

Shepsle, K. y Weingast, B. (1994). Positive theories of congressional institutions. Legislative Studies Quarterly, 19 (2), 149-179. Disponible en: https://doi.org/10.2307/440423.

Shugart, M. (2008). Comparative Executive-Legislative Relations. En R. A. W. Rhodes, S. Binder y B. Rockman (eds.). Oxford Handbook of Political Institutions (pp. 344-365). New York: Oxford University Press.

Siavelis, P. (2000). The President and Congress in Post-Authoritarian Chile: Institutional Constraints to Democratic Consolidation: University Park, PA: Pennsylvania University Press.

(2002). Coalitions, Voters and Party System Transformation in Post-Authoritarian Chile. Government and Opposition, 37 (1), 76-105. Disponible en: https://doi. org/10.1111/1477-7053.t01-1-00088.

- (2005). Electoral System, Coalitional Disintegration, and the Future of Chile's Concertacion. Latin American Research Review, 40 (1), 56-82. Disponible en: https://doi. org/10.1353/lar.2005.0014.

Sieberer, U. (2006). Party unity in parliamentary democracies: A comparative analysis. The Journal of Legislative Studies, 12 (2), 150-178. Disponible en: https://doi.org/10.1080/ 13572330600739413.

Tironi, E. y Agüero, F. (1999). ¿¿Sobrevivirá el nuevo paisaje político chileno? Estudios Públicos, 74, 151-168.

Toro Maureira, S. (2007). Conducta legislativa ante las iniciativas del Ejecutivo: unidad de los bloques políticos en Chile. Revista de Ciencia Politica (Santiago), 27 (1), 23-41. Disponible en: https://doi.org/10.4067/S0718-090X2007000200002.

— , Acevedo, C. y Matamala, K. (2011). Quebrando paradigmas en contextos presidencialistas: un examen sobre la capacidad legislativa en Chile. Revista Ibero-Americana de Estudios Legislativos, 1 (1), 102-110. Disponible en: https://doi.org/10.12660/riel. v1.n1.2010.4129.

- y Hurtado, N. (2016). The executive on the battlefield: government amendments and cartel theory in the Chilean Congress. The Journal of Legislative Studies, 22 (2), 196-215. Disponible en: https://doi.org/10.1080/13572334.2016.1163881. 
Valenzuela, S. (1999). Respuesta a Eugenio Tironi y Felipe Agüero. Reflexiones sobre el presente y futuro del paisaje político chileno a la luz de su pasado. Estudios Públicos, 75, 273-290.

Visconti, G. (2011). Comportamiento Diacrónico del Congreso en Chile. Revista de Ciencia Política, 31 (1), 91-115. Disponible en: https://doi.org/10.4067/S0718-090X2011000100005. 